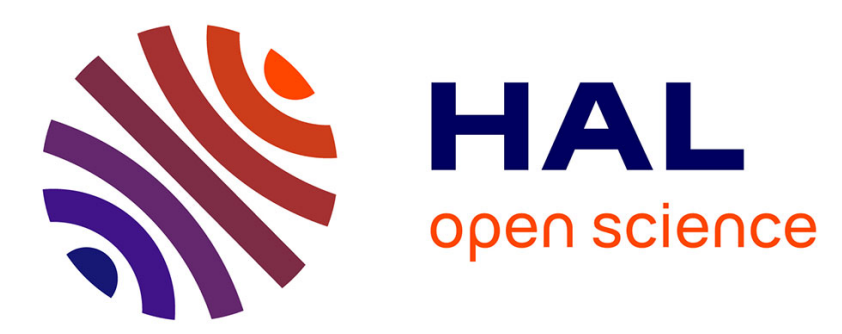

\title{
Asymptotic preserving schemes on distorted meshes for Friedrichs systems with sti relaxation: application to angular models in linear transport.
}

\author{
Christophe Buet, Bruno Després, Emmanuel Franck
}

\section{To cite this version:}

Christophe Buet, Bruno Després, Emmanuel Franck. Asymptotic preserving schemes on distorted meshes for Friedrichs systems with sti relaxation: application to angular models in linear transport.. Journal of Scientific Computing, 2015, 62 (issue 2), pp 371-398. 10.1007/s10915-014-9859-4 . hal00809444

\section{HAL Id: hal-00809444 \\ https://hal.science/hal-00809444}

Submitted on 9 Apr 2013

HAL is a multi-disciplinary open access archive for the deposit and dissemination of scientific research documents, whether they are published or not. The documents may come from teaching and research institutions in France or abroad, or from public or private research centers.
L'archive ouverte pluridisciplinaire HAL, est destinée au dépôt et à la diffusion de documents scientifiques de niveau recherche, publiés ou non, émanant des établissements d'enseignement et de recherche français ou étrangers, des laboratoires publics ou privés. 


\title{
Asymptotic preserving schemes on distorted meshes for Friedrichs systems with stiff relaxation: application to angular models in linear transport.
}

\author{
Christophe Buet, Bruno Després ${ }^{\dagger} \&$ Emmanuel Franck ${ }^{\ddagger}$
}

April 9, 2013

\begin{abstract}
In this paper we propose an asymptotic preserving scheme for a family of Friedrichs systems on unstructured meshes based on a decomposition between the hyperbolic heat equation and a linear hyperbolic which not involved in the diffusive regime. For the hyperbolic heat equation we use asymptotic preserving schemes recently designed in [FHSN11]-[BDF11]. To discretize the second part we use classical Rusanov or upwind schemes. To finish we apply this method for the discretization of the $P_{N}$ and $S_{N}$ models which are widely used in transport codes.
\end{abstract}

\section{Contents}

1 Introduction $\quad 2$

2 Friedrichs systems $\quad 3$

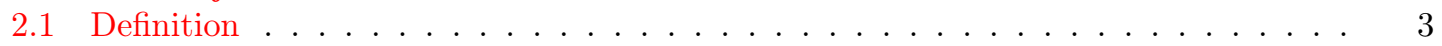

2.2 Diffusion limit of the Friedrichs systems . . . . . . . . . . . . . . . . . 4

3 Discretization strategy $\quad 6$

3.1 Principle of the "diffusive - non diffusive" decomposition . . . . . . . . . . . . . . 6

3.2 Asymptotic preserving scheme for hyperbolic heat equation . . . . . . . . . . . . 7

3.3 Numerical schemes for the hyperbolic "non-diffusive" part . . . . . . . . . . . . . . . . . . . 9

3.4 Structure of the algorithm . . . . . . . . . . . . . . . . . . . 10

4 Time discretizations $\quad 11$

4.1 Implicit discretization . . . . . . . . . . . . . . . . . . . . . . . . . . . . . . . . . . . . .

4.2 Semi-implicit schemes . . . . . . . . . . . . . . . . . . 11

5 Applications to the $P_{N}$ models $r$

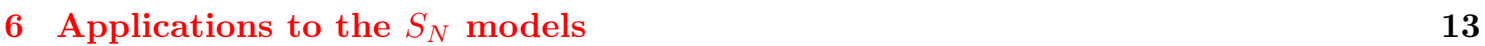

7 Numerical results $\quad 15$

7.1 The $P_{1}$ model . . . . . . . . . . . . . . . . . . . . . . . . 16

7.2 The $S_{2}$ model . . . . . . . . . . . . . . . . . . . . 17

7.2.1 Numerical results in the diffusion regime . . . . . . . . . . . . . . . . . 17

7.2 .2 Transport test case . . . . . . . . . . . . . . . . . . 18

7.3 The $P_{3}$ model . . . . . . . . . . . . . . . . . . . . . . . . . . 19

7.3.1 Numerical results for $P_{3}$ in diffusion limit . . . . . . . . . . . . . . . . 19

7.3.2 Fundamental solution for $P_{3}$ and $P_{1}$ models . . . . . . . . . . . . . . 21

7.3.3 Lattice problem for $P_{3}$ and $P_{1}$ models . . . . . . . . . . . . . . . . . 22

*CEA, DAM, DIF, F-91297 Arpajon Cedex

${ }^{\dagger}$ Laboratoire Jacques-Louis Lions, Université Pierre et Marie Curie, 75252 Paris Cedex 05, France

$\ddagger$ Max Planck Institute for Plasma Physics, Boltzmannstrasse 2 D-85748 Garching 


\section{Introduction}

We study the finite volume discretization of general linear hyperbolic systems with stiff source terms depending of a relaxation parameter $\varepsilon$, which admit an asymptotic diffusion limit. This type of system occurs in many physical applications (transport of particles, damped waves, electromagnetism, linearized gas dynamic, plasma physics) or in biology, and poses some numerical difficulties. The classical Godunov-type discretizations (upwind, Rusanov or HLL schemes) are not efficient because the time and spatial steps are constrained by the relaxation parameter $\varepsilon$ [BDF11], [JL96], [Jin99]. To treat this problem S. Jin, C. D. Levemore [JL96]-[Jin11] using the ideas of A. Y. Leroux [GL96], introduced the notion of asymptotic preserving schemes (AP schemes) which eliminate these constraints. To illustrate the advantage of asymptotic preserving discretizations, we propose a simple numerical example. We solve the hyperbolic heat equation

$$
\left\{\begin{array}{l}
\partial_{t} p+\frac{1}{\varepsilon} \partial_{x} u=0 \\
\partial_{t} u+\frac{1}{\varepsilon} \partial_{x} p+\frac{\sigma}{\varepsilon} u=0,
\end{array}\right.
$$

with two schemes: the upwind scheme and the asymptotic preserving scheme [GT01]. This model is approached when $\varepsilon$ is small by the following diffusion equation

$$
\partial_{t} p-\partial_{x}\left(\frac{1}{\sigma} \partial_{x} p\right)=0 .
$$

The initial data is given by $p(x, t=0)=G(x)$ with $G(x)$ a Gaussian function and $u(x, t=0)=0$. The parameters are given by $\sigma=1$ and $\varepsilon=0.001$. The time discretization is explicit and the time step is the half of the stability limit time step. The convergence errors are computed using the exact diffusion solution. The results proposed in table (1) and on figure (1) show that asymptotic
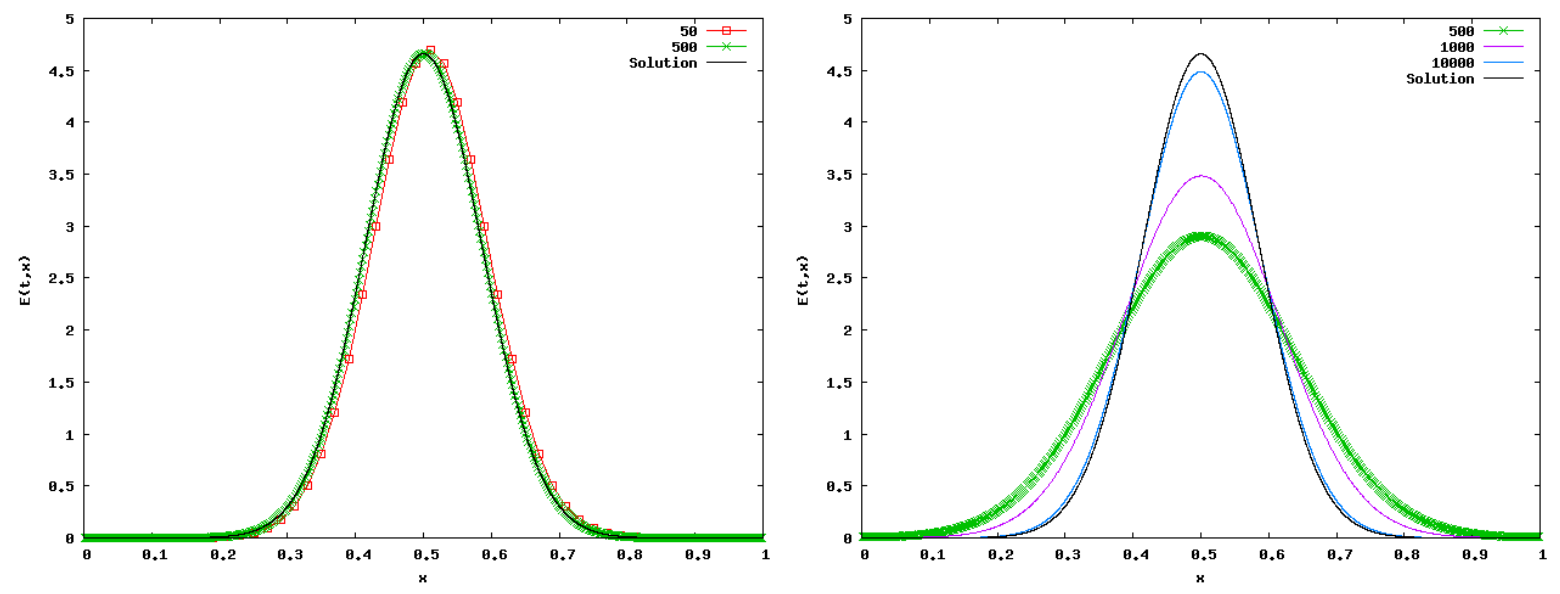

Figure 1: On the left: numerical solution of the Gosse-Toscani scheme for 50 and 500 cells, on the right: numerical solution of the upwind scheme 500, 1000 and 10000 cells

preserving scheme is more precise and cheaper in CPU time than the classical upwind scheme. These remarks may justify to use asymptotic preserving for this type of problem.

In 1D, many AP schemes have been designed: a non exhaustive list is S. Jin, C. D. Levermore [JL96] or L. Gosse, G. Toscani [GT01] for the hyperbolic heat equation, M. Lemou, L. Mieussens, N. Crouseilles [LM07]-[CL11]-[CL11] for some kinetic equations, C. Hauck, R. G. McClarren [HLMc10] for the $P_{N}$ equations, L. Gosse [Goss11], C. Buet and co-workers [BCLM02] or S. Jin and C. D. Levermore [JL91] for $S_{N}$ equations and C. Berthon, R. Turpault [BCT08]-[B010]-[BLeFT11]-[BT10] 


\begin{tabular}{|c|c|c|c|}
\hline Schemes & $L^{1}$ error & $L^{2}$ error & CPU time \\
\hline \hline AP scheme, 50 cells & 0.0065 & 0.0110 & $0 \mathrm{~m} 0.054 \mathrm{~s}$ \\
\hline AP scheme, 500 cells & 0.0001 & 0.00018 & $0 \mathrm{~m} 15.22 \mathrm{~s}$ \\
\hline upwind scheme, 500 cells & 0.445 & 0.647 & $0 \mathrm{~m} 24.317 \mathrm{~s}$ \\
\hline upwind scheme, 1000 cells & 0.279 & 0.113 & $2 \mathrm{~m} 9.530 \mathrm{~s}$ \\
\hline upwind scheme, 10000 cells & 0.0366 & 0.059 & $1485 \mathrm{~m} 4.26 \mathrm{~s}$ \\
\hline
\end{tabular}

Table 1: Table with numerical error and CPU time associated to the upwind and Gosse-Toscani schemes.

for generic systems and a non linear radiative transfer model.

For some applications (ICF simulations [DW94]) we are interested in, the stiff hyperbolic systems are coupled with Lagrangian hydrodynamic codes which generate very distorted meshes. Consequently it is important to design cell-centered asymptotic preserving schemes for the Friedrichs systems with a valid asymptotic diffusion limit on unstructured meshes. Currently these types of schemes based on the nodal scheme [BDF11]-[BDFCras]-[BDFproc] or the MPFA scheme [FHSN11][BM06]-[AE06] have been only designed for the hyperbolic heat equation and a non linear system used in radiative transfer.

The purpose of this paper is to extend Godunov-type asymptotic preserving schemes for the Friedrichs systems on unstructured meshes. Firstly we introduce the Friedrichs systems and give a formal proof of the existence of the diffusion limit. In the second part we define a numerical strategy based on a decomposition between a "diffusive" part similar to the hyperbolic heat equation and a "non diffusive" part which is negligible in the diffusion regime. This decomposition, close to the micro-macro decomposition [LM07] allows to design a very simple method to discretize stiff hyperbolic systems. Indeed, using an asymptotic preserving scheme for the "diffusive" part (nodal asymptotic preserving for example [BDF11]) and a classical hyperbolic scheme for the "non diffusive" part we obtain an asymptotic preserving discretization for the complete system. After this, we show how angular discretizations such as $P_{N}$ and $S_{N}$ models fall within this framework. This could be applied to other angular discretizations like those based on wavelet expansion for instance. To finish we propose some considerations on temporal discretizations and numerical results for $P_{N}$ and the $S_{N}$ systems.

\section{Friedrichs systems}

\subsection{Definition}

In this section we introduce linear Friedrichs systems with stiff source terms and their diffusion limit. We work in dimension two, $D \subset \mathbb{R}^{2}$ is a polygonal domain.

Definition 2.1. The sub-class of Friedrichs systems that we consider are defined by:

$$
\partial_{t} \mathbf{U}+\frac{1}{\varepsilon} A_{1} \partial_{x} \mathbf{U}+\frac{1}{\varepsilon} A_{2} \partial_{y} \mathbf{U}=-\frac{\sigma}{\varepsilon^{2}} R \mathbf{U},
$$

with $\mathbf{U}: D \times \mathbb{R}^{+} \longrightarrow \mathbb{R}^{n}, A_{1}, A_{2}, R$ are constant, symmetric and real square matrices. We assume moreover that the matrix $R$ is non negative, i.e. $(R x, x) \geq 0$ for all $x \in \mathbb{R}^{n}$ and $\operatorname{Ker} R \neq \emptyset$.

Non invertibility of the matrix $R$ is important to obtain a non trivial asymptotic regime. The parameter $\sigma$ is, in general, a positive and a lower bounded function but for the theoretical analysis we assume that $\sigma$ is constant and positive. The relaxation parameter is $\varepsilon \in] 0,1]$. Since the matrices $A_{1}, A_{2}$ are symmetric, the system is hyperbolic. Indeed the matrix $A_{1} n^{x}+A_{2} n^{y}$ is symmetric for all $\mathbf{n}=\left(n_{x}, n_{y}\right) \in \mathbb{S}^{1}$.

We define the functional spaces:

$$
L^{2}(D)=\left\{\mathbf{U},\|\mathbf{U}\|_{L^{2}(D)}=\left(\int_{D}(\mathbf{U}, \mathbf{U})\right)^{\frac{1}{2}} d x d y<\infty\right\},
$$




$$
H^{p}(D)=\left\{\mathbf{U} \in L^{2}(D),\|\mathbf{U}\|_{H^{p}(D)}=\sum_{a, b}^{a+b \leq p}\left\|\partial_{x^{a}, y^{b}} \mathbf{U}\right\|_{L^{2}}<\infty\right\},
$$

Foremost, we recall a classical result of stability for such systems.

Proposition 2.1. If $D=[0,1]^{2}$ with periodic boundary conditions, systems (2) are stable in $H^{p}(D)$.

Proof. We begin by proving the $L^{2}$ stability:

$$
\frac{1}{2} \frac{d}{d t}\|\mathbf{U}\|_{L^{2}(D)}^{2}=\int_{D}\left(\mathbf{U}, \partial_{t} \mathbf{U}\right) d x d y=-\frac{1}{\varepsilon} \int_{D}\left(A_{1} \partial_{x} \mathbf{U}+A_{2} \partial_{y} \mathbf{U}+\frac{\sigma}{\varepsilon} R \mathbf{U}, \mathbf{U}\right) d x d y .
$$

Since the matrix $A_{1}$ is constant and symmetric real, the first integral of the right hand side writes

$$
\int_{D}\left(A_{1} \partial_{x} \mathbf{U}, \mathbf{U}\right) d x d y=\frac{1}{2} \int_{D} \partial_{x}\left(\mathbf{U}, A_{1} \mathbf{U}\right) d x d y
$$

and since we consider periodic boundary conditions, $\int_{D}\left(A_{1} \partial_{x} \mathbf{U}, \mathbf{U}\right) d x d y=0$. In the same way we show that the second integral of the right hand side is null. Thus:

$$
\frac{1}{2} \frac{d}{d t}\|\mathbf{U}\|_{L^{2}(D)}^{2}=-\int_{D} \frac{\sigma}{\varepsilon^{2}}(R \mathbf{U}, \mathbf{U}) d x d y \leq 0
$$

since the matrix $R$ is non negative. The $L^{2}(D)$ norm of the solution decreases, thus the system is $L^{2}$-stable. We can check that $\mathbf{V}=\partial_{x^{a}} \partial_{y^{b}} \mathbf{U}$ is also a solution of the system (2) which gives the $H^{p}(D)$ stability.

\subsection{Diffusion limit of the Friedrichs systems}

In this section we propose a formal existence result for the asymptotic diffusion limit. We introduce a structure assumption.

Assumption $\left(H_{1}\right)$ : Let $\left(\mathbf{E}_{1}, \ldots \mathbf{E}_{n}\right)$ be the eigenvectors of $R$ and let $\left(\mathbf{E}_{1}, \ldots \mathbf{E}_{p}\right)$ be the basis of the kernel of $R$. The vectors are orthonormal. We assume that, there are two particular linearly independent eigenvectors $\mathbf{E}_{i_{1}}, \mathbf{E}_{i_{2}}$ associated to eigenvalues $\lambda_{i_{1}}>0, \lambda_{i_{2}}>0$ with the structure assumption

$$
\begin{cases}A_{1} \mathbf{E}_{i}=\gamma_{i}^{1} \mathbf{E}_{i_{1}} & \forall i \in\{1 \ldots p\}, \\ A_{2} \mathbf{E}_{i}=\gamma_{i}^{2} \mathbf{E}_{i_{2}} & \forall i \in\{1 \ldots p\} .\end{cases}
$$

In other sections we will show that the simplified models as $P_{N}$ or $S_{N}$ in linear transport theory satisfy the previous structure assumption. For the hyperbolic heat equation extended to $2 \mathrm{D} p=1$, $A_{1} \mathbf{E}_{1}=\mathbf{E}_{2}$ and $A_{2} \mathbf{E}_{1}=\mathbf{E}_{3}$ where $\mathbf{E}_{1}$ is the eigenvector associated to the eigenvalue 0 and $\mathbf{E}_{2}, \mathbf{E}_{3}$ are the eigenvectors associated at the eigenvalue 1 of the matrix $R$.

Proposition 2.2. If the assumption $\left(H_{1}\right)$ is satisfied, the system (2) admits the formal diffusion limit

$$
\partial_{t} \mathbf{V}-\frac{1}{\lambda_{i_{1}} \sigma} K_{1} \partial_{x x} \mathbf{V}-\frac{1}{\lambda_{i_{2}} \sigma} K_{2} \partial_{y y} \mathbf{V}=\mathbf{0},
$$

with $\mathbf{V}=\left(\left(\mathbf{U}, \mathbf{E}_{1}\right), \ldots .,\left(\mathbf{U}, \mathbf{E}_{p}\right)\right) \in \mathbb{R}^{p}, K_{1}=\gamma^{1} \otimes \gamma^{1} \in \mathbb{R}^{p} \times \mathbb{R}^{p}, K_{2}=\gamma^{2} \otimes \gamma^{2} \in \mathbb{R}^{p} \times \mathbb{R}^{p}$ non negatives symmetric matrices where the vectors $\gamma^{k}=\left(\gamma_{1}^{k}, \ldots, \gamma_{p}^{k}\right)$ are defined in (4).

Proof. Using a Hilbert expansion $\mathbf{U}=\mathbf{U}_{0}+\varepsilon \mathbf{U}_{1}+\varepsilon^{2} \mathbf{U}_{2}+o\left(\varepsilon^{2}\right)$ in (2), we obtain the hierarchy of equations:

$$
\begin{gathered}
\frac{1}{\varepsilon^{2}}: \quad R \mathbf{U}_{0}=\mathbf{0}, \\
\frac{1}{\varepsilon^{1}}: \quad A_{1} \partial_{x} \mathbf{U}_{0}+A_{2} \partial_{y} \mathbf{U}_{0}=-\sigma R \mathbf{U}_{1}, \\
\frac{1}{\varepsilon^{0}}: \quad \partial_{t} \mathbf{U}_{0}+A_{1} \partial_{x} \mathbf{U}_{1}+A_{2} \partial_{y} \mathbf{U}_{1}=-\sigma R \mathbf{U}_{2} .
\end{gathered}
$$


Equation (6) shows that $\mathbf{U}_{0} \in \operatorname{Ker} R$. Therefore,

$$
\mathbf{U}_{0}=\sum_{j=1}^{p}\left(\mathbf{U}, \mathbf{E}_{j}\right) \mathbf{E}_{j} .
$$

Equation (7) implies the existence of $\mathbf{U}_{1}$ up to an element of the kernel under the following compatibility condition

$$
A_{1} \partial_{x} \mathbf{U}_{0}+A_{2} \partial_{y} \mathbf{U}_{0} \in(\operatorname{Ker} R)^{\perp} .
$$

The assumption $\left(H_{1}\right)$ and the equation (9) show that $\mathbf{U}_{0}$ is such that

$$
A_{1} \partial_{x} \mathbf{U}_{0}=\left(\sum_{j}^{p} \partial_{x}\left(\mathbf{U}_{0}, \mathbf{E}_{j}\right) \gamma_{j}^{1}\right) \mathbf{E}_{i_{1}}, \quad A_{2} \partial_{y} \mathbf{U}_{0}=\left(\sum_{j}^{p} \partial_{y}\left(\mathbf{U}_{0}, \mathbf{E}_{j}\right) \gamma_{j}^{2}\right) \mathbf{E}_{i_{2}} .
$$

Using the definition of the eigenvectors and the linearity, we obtain the relation

$$
R\left(\frac{A_{1} \partial_{x} \mathbf{U}_{0}}{\lambda_{i_{1}}}+\frac{A_{2} \partial_{y} \mathbf{U}_{0}}{\lambda_{i_{2}}}\right)=A_{1} \partial_{x} \mathbf{U}_{0}+A_{2} \partial_{y} \mathbf{U}_{0},
$$

which gives the expression of $\mathbf{U}_{1}$

$$
\mathbf{U}_{1}=-\frac{1}{\sigma}\left(\frac{A_{1} \partial_{x} \mathbf{U}_{0}}{\lambda_{i_{1}}}+\frac{A_{2} \partial_{y} \mathbf{U}_{0}}{\lambda_{i_{2}}}\right)+\mathbf{z}, \quad, \mathbf{z} \in \operatorname{Ker} R
$$

Using the relation (8), we show the existence of $\mathbf{U}_{2}$ up to an element of the kernel under the following compatibility condition

$$
\partial_{t} \mathbf{U}_{0}+A_{1} \partial_{x} \mathbf{U}_{1}+A_{2} \partial_{y} \mathbf{U}_{1} \in(\operatorname{Ker} R)^{\perp} .
$$

Since $\operatorname{Ker}(R)=\operatorname{Vect}\left(\mathbf{E}_{1}, \ldots, \mathbf{E}_{p}\right)$, the compatibility condition can be written as

$$
\partial_{t}\left(\mathbf{U}_{0}, \mathbf{E}_{i}\right)+\partial_{x}\left(A_{1} \mathbf{U}_{1}, \mathbf{E}_{i}\right)+\partial_{y}\left(A_{2} \mathbf{U}_{1}, \mathbf{E}_{i}\right)=\mathbf{0} \quad i \in\{1 . . p\} .
$$

Now we plug the relation (10) in (11) to obtain the equations

$$
\left\{\begin{array}{l}
\partial_{t}\left(\mathbf{U}_{0}, \mathbf{E}_{i}\right)-\frac{1}{\lambda_{i_{1}} \sigma} \partial_{x x}\left(A_{1} \mathbf{U}_{0} A_{1} \mathbf{E}_{i}\right)-\frac{1}{\lambda_{i_{2}} \sigma} \partial_{y y}\left(A_{2} \mathbf{U}_{0}, A_{2} \mathbf{E}_{i}\right) \\
-\frac{1}{\lambda_{i_{2}} \sigma} \partial_{x y}\left(A_{1} \mathbf{U}_{0}, A_{2} \mathbf{E}_{i}\right)-\frac{1}{\lambda_{i_{1}} \sigma} \partial_{y x}\left(A_{2} \mathbf{U}_{0}, A_{1} \mathbf{E}_{i}\right)+N_{i}=0, \text { for } i \in\{1 . . p\}
\end{array}\right.
$$

where

$$
N_{i}=\partial_{x}\left(A_{1} \mathbf{z}, \mathbf{E}_{i}\right)+\partial_{y}\left(A_{2} \mathbf{z}, \mathbf{E}_{i}\right)=\partial_{x}\left(\mathbf{z}, A_{1} \mathbf{E}_{i}\right)+\partial_{y}\left(\mathbf{z}, A_{2} \mathbf{E}_{i}\right)
$$

The assumption $\left(H_{1}\right)$ and the orthogonality of the eigenvectors show that the terms $A_{1} \mathbf{E}_{i}, A_{2} \mathbf{E}_{i}$ are orthogonal to $\mathbf{z}$. Consequently the terms $N_{i}$ (13) are equal to zero. Now we study the cross terms $\left(A_{1} \mathbf{U}_{0}, A_{2} \mathbf{E}_{i}\right)$ and $\left(A_{2} \mathbf{U}_{0}, A_{1} \mathbf{E}_{i}\right)$. One has

$$
\begin{aligned}
& \left(A_{1} \mathbf{U}_{0}, A_{2} \mathbf{E}_{i}\right)=\left(A_{1}\left(\sum_{j=1}^{p}\left(\mathbf{U}_{0}, \mathbf{E}_{j}\right) \mathbf{E}_{j}\right), A_{2} \mathbf{E}_{i}\right)=\left(\left(\sum_{j=1}^{p} \gamma_{j}^{1}\left(\mathbf{U}_{0}, \mathbf{E}_{j}\right)\right) \mathbf{E}_{i_{1}}, \gamma_{i}^{2} \mathbf{E}_{i_{2}}\right)=0, \\
& \left(A_{2} \mathbf{U}_{0}, A_{1} \mathbf{E}_{i}\right)=\left(A_{2}\left(\sum_{j=1}^{p}\left(\mathbf{U}_{0}, \mathbf{E}_{j}\right) \mathbf{E}_{j}\right), A_{1} \mathbf{E}_{i}\right)=\left(\left(\sum_{j=1}^{p} \gamma_{j}^{2}\left(\mathbf{U}_{0}, \mathbf{E}_{j}\right)\right) \mathbf{E}_{i_{2}}, \gamma_{i}^{1} \mathbf{E}_{i_{1}}\right)=0 .
\end{aligned}
$$

The cross terms vanish. For the other terms we obtain

$$
\left(A_{k} \mathbf{U}, A_{k} \mathbf{E}_{i}\right)=\sum_{j}\left(\mathbf{U}_{0}, \mathbf{E}_{j}\right)\left(A_{k} \mathbf{E}_{j}, A_{k} \mathbf{E}_{i}\right)=\sum_{j}\left(\mathbf{U}_{0}, \mathbf{E}_{j}\right) \gamma_{j}^{k} \gamma_{i}^{k} .
$$

So the equations (12) with $\mathbf{U}_{0}=\mathbf{U}$ are equivalent to the equations (2) with $K_{1}=\gamma^{1} \otimes \gamma^{1}$ and $K_{2}=\gamma^{2} \otimes \gamma^{2}$. These matrices are symmetric by definition. Moreover

$$
\left(\mathbf{X}, K_{k} \mathbf{X}\right)=\left(\gamma^{k}, \mathbf{X}\right)^{2} \geq 0 \forall \mathbf{X} \in \mathbb{R}^{p},
$$

therefore the matrices $K_{k}$ are non negatives. 
Remark 2.3. - Since the matrices $K_{1}$ and $K_{2}$ are non negatives, the system (5) is dissipative.

- The size of the diffusion equation (5) is equal at the multiplicity of the eigenvalue 0 of the matrix $R$.

- The hypothesis $\left(H_{1}\right)$ is sufficient but not necessary. The assumption $A_{k} \mathbf{E}_{i} \in(\operatorname{Ker} R)^{\perp}$ for $i \in\{1 . . p\}$ is also possible.

- If $\lambda_{i_{1}}=\lambda_{i_{2}}$ the diffusion equation is isotropic.

- In $3 D$ the proof uses the same principle.

\section{Discretization strategy}

In this section, we propose a strategy to design finite volume schemes valid for Friedrichs system on unstructured meshes. The method is only valid for the Friedrichs systems which have a scalar diffusion limit ( $\operatorname{dim} \operatorname{Ker} R=1$ ). The idea is to split the Friedrichs system between a "diffusive" part similar to the hyperbolic heat equation and a "non diffusive" part which is negligible in the diffusive regime. This method is in the principle very close to the micro-macro decomposition used in [LM07]-[CL11].

\subsection{Principle of the "diffusive - non diffusive" decomposition}

The "diffusive - non diffusive" decomposition uses the particular structure of some Friedrichs systems described by the following assumption. Assumption $\left(\mathrm{H}_{2}\right)$ : Assume that

$$
\left(H_{2}\right)\left\{\begin{array}{l}
\operatorname{dim}(\operatorname{Ker} R)=1, \text { consequently } \operatorname{Ker} R=\operatorname{Vect}\left(\mathbf{E}_{1}\right), \\
\lambda_{2}=\lambda_{3}=\lambda \text { (we study isotropic diffusion limit), } \\
A_{1} \mathbf{E}_{1}=a \mathbf{E}_{2}, A_{2} \mathbf{E}_{1}=a \mathbf{E}_{3},
\end{array}\right.
$$

with $\lambda_{i}$ the eigenvalues, by convention $\lambda_{1}=0$, and $\mathbf{E}_{i}$ the eigenvectors of $R$.

Since $R$ is symmetric the matrix can be written on the following form $R=Q D Q^{t}$ with $D$ diagonal matrix and $Q$ an orthogonal matrix where the column are the eigenvectors of $R$. Since $R$ is non negative, the coefficients of $D$ are non negative. We define $\mathbf{V}=Q^{t} \mathbf{U}$,

$$
\partial_{t} \mathbf{V}+\frac{1}{\varepsilon}\left(A_{1}^{\prime} \partial_{x} \mathbf{V}+A_{2}^{\prime} \partial_{y} \mathbf{V}\right)=-\frac{\sigma}{\varepsilon^{2}} D \mathbf{V}
$$

with $A_{1}^{\prime}=Q^{t} A_{1} Q, A_{2}^{\prime}=Q^{t} A_{2} Q$ symmetric matrices.

Lemma 3.1. Under the assumption $\left(H_{2}\right)$, the matrices $A_{1}^{\prime}, A_{2}^{\prime}$ have the following block structure

$$
A_{1}^{\prime}=\left(\begin{array}{cc}
0 & C_{1} \\
C_{1}^{t} & B_{1}
\end{array}\right) \quad A_{2}^{\prime}=\left(\begin{array}{cc}
0 & C_{2} \\
C_{2}^{t} & B_{2}
\end{array}\right) .
$$

where $B_{1}$ and $B_{2}$ are $(n-1) \times(n-1)$ symmetric matrices, and $C_{1}$ and $C_{2}$ are $1 \times(n-1)$ matrices whose elements are defined by $C_{k}, j=a \delta_{k, j}$ for $k=1,2$ and $j=1, \ldots, n-1$ and $\delta_{k, j}$ stands for the Kronecker product.

Proof. Let us consider the matrix $A_{1}^{\prime}$. Using the definition of the matrix $Q$ we have

$$
A_{1, i j}^{\prime}=\left(\mathbf{E}_{i}, A_{1} \mathbf{E}_{j}\right) .
$$

For the first line we have then, remembering that $E_{1}, \ldots, E_{n}$ is an orthonormal basis,

$$
A_{1,1 j}^{\prime}=\left(\mathbf{E}_{1}, A_{1} \mathbf{E}_{j}\right)=\left(A_{1} \mathbf{E}_{1}, \mathbf{E}_{j}\right)=\left(\mathbf{E}_{1}, A_{1} \mathbf{E}_{j}\right)=\left(a \mathbf{E}_{2}, \mathbf{E}_{j}\right)=a \delta_{2 j} .
$$

Since we are dealing with symmetric matrices, the results follows for the first column. By the same way we obtain the desired result for the matrix $A_{2}^{\prime}$. 
Therefore we can rewrite the system (18) as

$$
\partial_{t} \mathbf{V}+\frac{1}{\varepsilon}\left(P_{1, x} \partial_{x} \mathbf{V}+P_{1, y} \partial_{y} \mathbf{V}\right)+\frac{1}{\varepsilon}\left(A_{1}^{\prime \prime} \partial_{x} \mathbf{V}+A_{2}^{\prime \prime} \partial_{y} \mathbf{V}\right)=-\frac{\sigma}{\varepsilon^{2}} D \mathbf{V}
$$

where the matrices $P_{1, x}, P_{1, y}$ are defined, as block matrices, by

$$
P_{1, x}=\left(\begin{array}{cc}
Q_{1} & 0 \\
0 & 0
\end{array}\right) \quad P_{1, y}=\left(\begin{array}{cc}
Q_{2} & 0 \\
0 & 0
\end{array}\right)
$$

where $Q_{1}$ and $Q_{2}$ are the $3 \times 3$ matrices

$$
Q_{1}=\left(\begin{array}{ccc}
0 & a & 0 \\
a & 0 & 0 \\
0 & 0 & 0
\end{array}\right) \quad Q_{2}=\left(\begin{array}{ccc}
0 & 0 & a \\
0 & 0 & 0 \\
a & 0 & 0
\end{array}\right)
$$

and $A_{1}^{\prime \prime}=A_{1}^{\prime}-P_{1, x}, A_{2}^{\prime \prime}=A_{2}^{\prime}-P_{1, y}$ are symmetric matrices where the first line and column of the matrices $A_{1}^{\prime \prime}, A_{2}^{\prime \prime}$ are equal to zero. Next we decompose the model (19) between two systems. The first part of the system is very close to the hyperbolic heat equation

$$
\partial_{t} \mathbf{V}+\frac{1}{\varepsilon}\left(P_{1, x} \partial_{x} \mathbf{V}+P_{1, y} \partial_{y} \mathbf{V}\right)=-\frac{\sigma}{\varepsilon^{2}} D^{\prime} \mathbf{V}
$$

with, for the diagonal matrix $D^{\prime}, D_{11}^{\prime}=0, D_{22}^{\prime}=D_{33}^{\prime}=\lambda_{2}$ and $D_{i i}^{\prime}=0 i \geq 4$. The second system is given by

$$
\partial_{t} \mathbf{V}+\frac{1}{\varepsilon}\left(A_{1}^{\prime \prime} \partial_{x} \mathbf{V}+A_{2}^{\prime \prime} \partial_{y} \mathbf{V}\right)=-\frac{\sigma}{\varepsilon^{2}} D^{\prime \prime} \mathbf{V}
$$

with, for the diagonal matrix $D^{\prime \prime}, D_{11}^{\prime \prime}=D_{22}^{\prime \prime}=D_{33}^{\prime \prime}=0$ and $D_{i i}^{\prime \prime}=\lambda_{i} i \geq 4$. This decomposition is a little bit different from the micro-macro decomposition. Indeed when we use the micro-macro decomposition for the linear kinetic equation (some Friedrichs systems can be interpreted as angular discretization to the linear kinetic equation) we split the isotropic part homogeneous to $O(1)$ and the residual homogeneous to $O(\varepsilon)$. When we apply an asymptotic analysis to our decomposition we remark that we split the equations associated to the unknowns homogeneous to $O(1)$ and $O(\varepsilon)$ (first system) which gives the diffusion limit and the equations associated to the unknowns homogeneous to $O\left(\varepsilon^{2}\right)$ (second system) which are negligible in the diffusion regime.

Principle of discretization:

The proposed numerical method consists to use an asymptotic preserving scheme for the "diffusive" part (20) and a classical hyperbolic scheme for the "non diffusive" part (21). In the following section we will introduce the different numerical schemes for the two parts of the decomposition.

\subsection{Asymptotic preserving scheme for hyperbolic heat equation}

The discretization of the "diffusive" part (20) is based on a specific asymptotic preserving scheme that we recall now for the hyperbolic heat equation

$$
\left\{\begin{array}{c}
\partial_{t} p+\frac{a}{\varepsilon} \operatorname{div} \mathbf{u}=0 \\
\partial_{t} \mathbf{u}+\frac{a}{\varepsilon} \nabla p=-\frac{\sigma \lambda}{\varepsilon^{2}} \mathbf{u}
\end{array}\right.
$$

where $p \in \mathbb{R}$ and $\mathbf{u} \in \mathbb{R}^{2}$. In [BDF11] we have observed that the classical extension of Godunov-type asymptotic preserving schemes (Jin-Levermore scheme [JL96] or Gosse-Toscani [GT01] scheme) in 2D are convergent only on regular meshes which satisfy the Delaunay condition [EGH00]. Indeed the numerical viscosity of the hyperbolic scheme gives a non consistent limit diffusion scheme (two point flux approximation (TPFA) scheme [BDF11]-[EGH00]) on unstructured meshes. To solve this problem two methods have been proposed. In [BDF11] the extensions of Jin-Levermore scheme and Gosse-Toscani scheme have been designed using the nodal finite volumes formulation (the fluxes are localized at the nodes) [KD10]-[CDDL09] because the numerical viscosity of this 
scheme has a better structure. Another method is introduced in [FHSN11] based on the convergent diffusion scheme MPFA (MultiPoint Flux Approximation) [AE06].

Let us consider an unstructured mesh in dimension two. The mesh is defined by a finite number of vertices $\mathbf{x}_{r}$ and cells $\Omega_{j}$. We denote $\mathbf{x}_{j}$ a point arbitrarily chosen inside $\Omega_{j}$. For simplicity we will call this point the center of the cell. By convention the vertices are listed counter-clockwise $\mathbf{x}_{r-1}, \mathbf{x}_{r}, \mathbf{x}_{r+1}$ with coordinates $\mathbf{x}_{r}=\left(x_{r}, y_{r}\right)$. The length $l_{j r}$ and the normal $\mathbf{n}_{j r}$ associated to the node $r$ et the cell $j$ are defined by

$$
l_{j r}=\frac{1}{2}\left|\mathbf{x}_{r+1}-\mathbf{x}_{r-1}\right| \text { and } \mathbf{n}_{j r}=\frac{1}{2 l_{j r}}\left(\begin{array}{c}
-y_{r-1}+y_{r+1} \\
x_{r-1}-x_{r+1}
\end{array}\right) .
$$

The convention is that the norm of a vector $\mathbf{x} \in \mathbb{R}^{2}$ is denoted as $|\mathbf{x}|$. The scalar product of two vectors is $(\mathbf{x}, \mathbf{y})$. The JL-(b) nodal-AP scheme (2-D extension of the Gosse-Toscani scheme) writes,

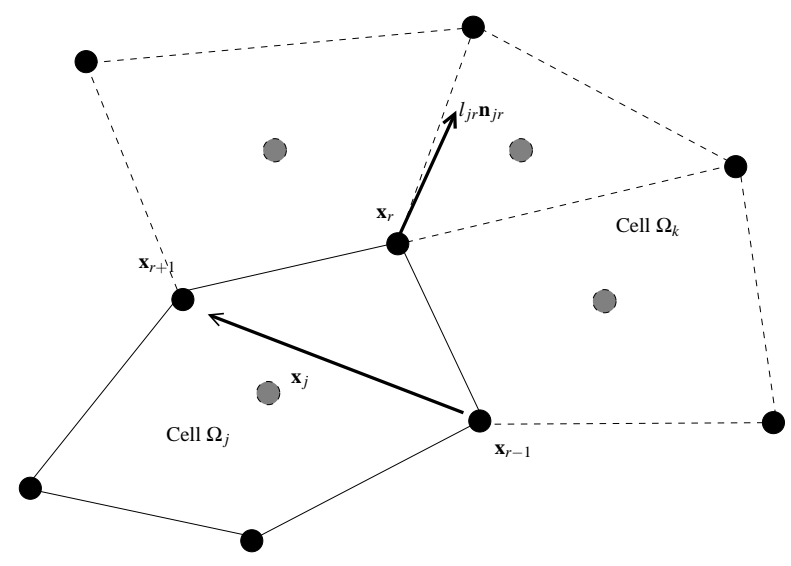

Figure 2: Notation for node formulation. The corner length $l_{j r}$ and the corner normal $\mathbf{n}_{j r}$ are defined in equation (23). Notice that $l_{j r} \mathbf{n}_{j r}$ is equal to the orthogonal vector to the half of the vector that starts at $\mathbf{x}_{r-1}$ and finish at $\mathbf{x}_{r+1}$. The center of the cell is an arbitrary point inside the cell.

see [BDF11],

$$
\left\{\begin{array}{l}
\left|\Omega_{j}\right| \partial_{t} p_{j}+\frac{a}{\varepsilon} \sum_{r}\left(l_{j r} M_{r} \mathbf{u}_{r}, \mathbf{n}_{j r}\right)=0 \\
\left|\Omega_{j}\right| \partial_{t} \mathbf{u}_{j}+\frac{a}{\varepsilon} \sum_{r} \widehat{\alpha}_{j r} M_{r}\left(\mathbf{u}_{j}-\mathbf{u}_{r}\right)=-\frac{1}{\varepsilon}\left(\sum_{r} \widehat{\alpha}_{j r}\left(\widehat{I}_{d}-M_{r}\right)\right) \mathbf{u}_{j},
\end{array}\right.
$$

with the fluxes

$$
\left(\sum_{j} \widehat{\alpha}_{j r}\right) \mathbf{u}_{r}=\sum_{j} l_{j r} p_{j} \mathbf{n}_{j r}+\sum_{j} \widehat{\alpha}_{j r} \mathbf{u}_{j}, \quad M_{r}=\left(\sum_{j} \widehat{\alpha}_{j r}+\frac{\sigma \lambda}{a \varepsilon} \sum_{j} \widehat{\beta}_{j r}\right)^{-1}\left(\sum_{j} \widehat{\alpha}_{j r}\right)
$$

and the tensors

$$
\widehat{\beta}_{j r}=l_{j r} \mathbf{n}_{j r} \otimes\left(\mathbf{x}_{r}-\mathbf{x}_{j}\right), \quad \widehat{\alpha}_{j r}=l_{j r} \mathbf{n}_{j r} \otimes \mathbf{n}_{j r} .
$$

This scheme admits the following limit diffusion scheme on coarse grids

$$
\left\{\begin{array}{l}
\left|\Omega_{j}\right| \partial_{t} p_{j}(t)+\frac{a^{2}}{\sigma \lambda} \sum_{r} l_{j r}\left(\mathbf{u}_{r}, \mathbf{n}_{j r}\right)=0 \\
\left(\sum_{j} l_{j r} \mathbf{n}_{j r} \otimes\left(\mathbf{x}_{r}-\mathbf{x}_{j}\right)\right) \mathbf{u}_{r}=\sum_{j} l_{j r} p_{j} \mathbf{n}_{j r}
\end{array}\right.
$$

We recall some properties of this scheme: 
- The scheme (24) is stable for the $L^{2}$ norm [BDF11].

- The matrix $A_{r}=\sum_{j} l_{j r} \mathbf{n}_{j r} \otimes\left(\mathbf{x}_{r}-\mathbf{x}_{j}\right)$ is positive and coercive under non restrictive conditions on the meshes.

- In [BDF11] we prove that the limit diffusion scheme is convergent if the matrix $A_{r}$ is coercive.

- If we implicit the source term of (24), we observe numerically that the stability CFL condition is independent of $\varepsilon$.

- These schemes exhibit spurious mods [BDF11]-[CDDL09] which degrade the quality of the numerical solution. A solution, based on geometrical corrections, to treat this problem is proposed in [BDF11].

- Numerical tests show convergence in all cases.

\subsection{Numerical schemes for the hyperbolic "non-diffusive" part}

We discretize the "non-diffusive" part (21) using a classical hyperbolic scheme. First of all, we recall two different schemes, the upwind scheme and the Rusanov scheme. The upwind scheme in dimension two has been studied in [Cou06]. Consider

$$
\left\{\begin{array}{l}
\partial_{t} \mathbf{U}+M_{1} \partial_{x} \mathbf{U}+M_{2} \partial_{y} \mathbf{U}=\mathbf{0} \\
\mathbf{U}(t=0)=\mathbf{U}_{0}
\end{array}\right.
$$

with two arbitrary real symmetric matrices $M_{1}$ and $M_{2}$.

For a cell of index $j, \mathbf{n}_{j k}$ denotes the outward normal associated to the interface $\partial \Omega_{j k}$ between the cell $j$ and one of its neighbors of index $k, G_{j k}=M_{1} n_{j k}^{x}+M_{2} n_{j k}^{y}=-G_{k j}$ and $l_{j k}=\left|\partial \Omega_{j k}\right|$.

Definition 3.1. The space discretization of the upwind scheme is

$$
\left|\Omega_{j}\right| \partial_{t} \mathbf{U}_{j}(t)+\sum_{k} l_{j k} \mathbf{U}_{j k}=\mathbf{0},
$$

with the fluxes

$$
\mathbf{U}_{j k}=\left(G_{j k}\right)^{+} \mathbf{U}_{j}+\left(G_{j k}\right)^{-} \mathbf{U}_{k},
$$

where the matrices $\left(G_{j k}\right)^{+,-}$are the non positive and the non negative parts of the matrix defined by $G_{j k}^{+,-}=P^{-1} D^{+,-} P$ with $D^{+,-}$the matrices of the positive and negative eigenvalues of $G_{j k}$ and $P$ is the orthogonal matrix such that $P G_{j k} P^{-1}$ is diagonal.

The computation cost associated to the upwind scheme can be important for large linear system. Therefore we propose another choice: the Rusanov scheme. This scheme use only an estimation of the maximal eigenvalue.

Definition 3.2. The Rusanov scheme is defined by

$$
\left|\Omega_{j}\right| \partial_{t} \mathbf{U}_{j}+\sum_{k} l_{j k} \mathbf{U}_{j k}=\mathbf{0}
$$

with the numerical fluxes given by

$$
\mathbf{U}_{j k}=G_{j k} \frac{\mathbf{U}_{j}+\mathbf{U}_{k}}{2}+S_{j k} \frac{\mathbf{U}_{j}-\mathbf{U}_{k}}{2},
$$

and the local speed $S_{j k}$ is such that $S_{j k} \geq \max _{i}\left(\lambda_{j k}^{i}\right)$ and $\lambda_{j k}^{i}$ are the eigenvalues of $G_{j k}$. 


\subsection{Structure of the algorithm}

We are now ready to recapitulate the explicit version of the proposed "diffusive-non diffusive" (19) decomposition.

\section{Algorithm 3.2.}

- Preparation

- Step 1: We diagonalize in the basis of $R$ the system

$$
\partial_{t} \mathbf{U}+\frac{1}{\varepsilon} A_{1} \partial_{x} \mathbf{U}+\frac{1}{\varepsilon} A_{2} \partial_{y} \mathbf{U}=-\frac{\sigma}{\varepsilon^{2}} R \mathbf{U}
$$

- Step 2: We decompose the diagonal system

$$
\partial_{t} \mathbf{V}+\frac{1}{\varepsilon} A_{1}^{\prime} \partial_{x} \mathbf{V}+\frac{1}{\varepsilon} A_{2}^{\prime} \partial_{y} \mathbf{V}=-\frac{\sigma}{\varepsilon^{2}} D \mathbf{V}
$$

with $\mathbf{V}=Q^{t} \mathbf{U}, A_{1}^{\prime}=Q^{t} A_{1} Q$ et $A_{2}^{\prime}=Q^{t} A_{2} Q$. We obtain

$$
\partial_{t} \mathbf{V}+\frac{1}{\varepsilon}\left(P_{1, x} \partial_{x} \mathbf{V}+P_{1, y} \partial_{y} \mathbf{V}\right)+\frac{1}{\varepsilon}\left(A_{1}^{\prime \prime} \partial_{x} \mathbf{V}+A_{2}^{\prime \prime} \partial_{y} \mathbf{V}\right)=-\frac{\sigma}{\varepsilon^{2}} D \mathbf{V}
$$

- Step 3: The system homogeneous to the hyperbolic heat equation is

$$
\partial_{t} \mathbf{V}+\frac{1}{\varepsilon}\left(P_{1, x} \partial_{x} \mathbf{V}+P_{1, y} \partial_{y} \mathbf{V}\right)=-\frac{\sigma}{\varepsilon^{2}} D^{\prime} \mathbf{V}
$$

Using an asymptotic preserving discretization such as the JL-(b) (24)-(25) scheme or the $P_{1}-M P F A$ scheme [FHSN11], we define a matrix $P_{h}$ such that

$$
\mathbf{V}_{h}^{n+1}=\mathbf{V}_{h}^{n}+\Delta t P_{h} \mathbf{V}_{h}^{n}
$$

is an explicit discretization of (35).

- Step 4: The second system is

$$
\partial_{t} \mathbf{V}+\frac{1}{\varepsilon}\left(A_{1}^{\prime \prime} \partial_{x} \mathbf{V}+A_{2}^{\prime \prime} \partial_{y} \mathbf{V}\right)=-\frac{\sigma}{\varepsilon^{2}} D^{\prime \prime} \mathbf{V}
$$

Using a standard finite volume scheme such as Rusanov (30)-(31) or upwind (28)-(29), we define a matrix $A_{h}$ such that

$$
\mathbf{V}_{h}^{n+1}=\mathbf{V}_{h}^{n}+\Delta t A_{h} \mathbf{V}_{h}^{n}
$$

is an explicit discretization of (37).

- Loop in time

- Step 1: $\mathbf{V}_{h}^{n}=Q^{t} \mathbf{U}_{h}^{n}$

- Step 2: We apply the explicit scheme

$$
\mathbf{V}_{h}^{n+1}=\mathbf{V}_{h}^{n}+\Delta t\left(P_{h}+A_{h}\right) \mathbf{V}_{h}^{n}
$$

- Step 3: $\mathbf{U}_{h}^{n+1}=Q \mathbf{V}_{h}^{n+1}$

Remark 3.3. In this work we diagonalize the system to obtain the primitive variables at each time step. However it is not necessary, we can diagonalize the system only at the first time step.

Remark 3.4. Usual boundary conditions are easy to incorporate in (39) with standard technics such as the ghost cells method. 


\section{Time discretizations}

Now we quote some remarks about the time discretization. The stability condition of the time scheme associated to the "diffusive - non diffusive" decomposition is given by the stability conditions of each part of the decomposition. Some asymptotic preserving schemes used for the "diffusive" part and classical schemes used for the "non diffusive" part admit CFL conditions dependent of $\varepsilon$. To treat this problem, we can use a fully implicit scheme or design semi-implicit scheme stable on the CFL condition independent of $\varepsilon$ [BDF11]. Firstly we will study the implicit discretization of the "diffusive - non diffusive" decomposition.

\subsection{Implicit discretization}

We study the $L^{2}$ stability of the implicit of the algorithm (39). The standard $L^{2}$ norm is $\|\mathbf{V}\|_{L^{2}}^{2}=$ $\sum_{j}\left|\Omega_{j}\right|\left(\mathbf{V}_{j}, \mathbf{V}_{j}\right)$ and the scalar product is $(\mathbf{U}, \mathbf{V})=\sum_{j}\left|\Omega_{j}\right|\left(\mathbf{U}_{j}, \mathbf{V}_{j}\right)$. Let us assume for simplicity that periodic boundary conditions are used so that $\left(\mathbf{X}, P_{h} \mathbf{X}\right) \leq 0$ for all $\mathbf{X} \in \mathbb{R}^{n \times n_{c}}$ and $n_{c}$ is the number of cells: this is proved in [BDF11] for the JL-(b) scheme. Moreover one has $\left(\mathbf{X}, A_{h} \mathbf{X}\right) \leq 0$ for standard upwind discretizations.

Proposition 4.1. The implicit scheme

$$
M \mathbf{V}_{h}^{n+1}=M \mathbf{V}_{h}^{n}+\Delta t P_{h} \mathbf{V}_{h}^{n+1}+\Delta t A_{h} \mathbf{V}_{h}^{n+1}
$$

is stable in the norm $L^{2}(D)$.

Proof. By multiplying (40) by $\mathbf{V}_{h}^{n+1}$ we obtain

$$
\left(M \mathbf{V}_{h}^{n+1}, \mathbf{V}_{h}^{n+1}\right)=\left(M \mathbf{V}_{h}^{n}, \mathbf{V}_{h}^{n+1}\right)+\Delta t\left(P_{h} \mathbf{V}_{h}^{n+1}, \mathbf{V}_{h}^{n+1}\right)+\Delta t\left(A_{h} \mathbf{V}_{h}^{n+1}, \mathbf{V}_{h}^{n+1}\right) .
$$

To conclude, using the inequalities $\left(\mathbf{V}_{h}^{n+1}, P_{h} \mathbf{V}_{h}^{n+1}\right) \leq 0,\left(\mathbf{V}_{h}^{n+1}, A_{h} \mathbf{V}_{h}^{n+1}\right) \leq 0$ and the CauchySchwartz inequality we obtain

$$
\left\|\mathbf{V}^{n+1}\right\|_{L^{2}} \leq\left\|\mathbf{V}^{n}\right\|_{L^{2}}
$$

\subsection{Semi-implicit schemes}

We design semi-implicit schemes modifying the "diffusive - non diffusive" decomposition to obtain a restrictive-less CFL. We study the scheme for the "diffusive" part. In 1D the JL-(b) scheme (24) which is equivalent to the Gosse-Toscani scheme is stable for the $L^{\infty}$ norm under the CFL condition [BDF11]:

$$
\Delta t\left(\frac{M}{\varepsilon h}+\frac{M \sigma}{\varepsilon^{2}}\right) \leq 1,
$$

with $M=\frac{2 \varepsilon}{2 \varepsilon+\sigma h}$. The previous CFL condition is equivalent to

$$
\Delta t\left(\frac{1}{\varepsilon h}\right) \leq 1 .
$$

If we use a local-implicit discretization for the source term we obtain

$$
\Delta t\left(\frac{1}{\varepsilon h+\frac{h^{2}}{\sigma}}\right) \leq 1
$$

A reasonable CFL condition is the sum of the classical hyperbolic CFL condition and the parabolic CFL condition. These remarks show that we can obtain a stability condition independent of $\varepsilon$ for the "diffusive" part using the semi-implicit JL-(b) scheme (extension in 2D of the Gosse-Toscani scheme). However, for the "non diffusive" part the CFL condition of semi-implicit scheme is close to (41) in 1D. Therefore we propose to multiply the Rusanov or upwind fluxes by an adapted factor $M$ in the "non diffusive" part and use a local implicit discretization of the source term. This 
strategy allows to obtain CFL condition close to (42) for the complete system.

The factor $M$ depends on hyperbolic system studied and the scheme used. For the Rusanov scheme where the Rusanov velocity is $S_{j k}$ and $\frac{1}{c_{o} \sigma}$ the diffusion coefficient, the factor $M$ is defined by

$$
M_{j k}=\frac{2 S_{j k} \varepsilon}{2 S_{j k} \varepsilon+c_{o} \sigma_{j k} h},
$$

with $h$ a quantity homogeneous to the characteristic length of the mesh. For example we can use $h=d\left(\mathbf{x}_{j}, \mathbf{x}_{k}\right)$ with $\mathbf{x}_{j}, \mathbf{x}_{k}$ the center of the cells.

For the upwind scheme with a velocity $\lambda_{j k}$ and the same diffusion coefficient $M_{j k}$ is defined by

$$
M_{j k}=\frac{2 \lambda_{j k} \varepsilon}{2 \lambda_{j k} \varepsilon+c_{o} \sigma_{j k} h}
$$

\section{Applications to the $P_{N}$ models}

The transport of some type of particles is described by the following transport equation with scattering term (for example: radiative transfer equation, neutron transport linear equation)

$$
\partial_{t} f(\mathbf{x}, \boldsymbol{\Omega}, t)+\frac{1}{\varepsilon} \boldsymbol{\Omega} . \nabla f(\mathbf{x}, \boldsymbol{\Omega}, t)=\frac{\sigma}{\varepsilon^{2}} \int_{\mathbb{S}^{2}}\left(f\left(\mathbf{x}, \boldsymbol{\Omega}^{\prime}, t\right)-f(\mathbf{x}, \boldsymbol{\Omega}, t)\right) d \mathbf{\Omega}^{\prime} .
$$

The $P_{N}$ systems are obtained expanding the equation (43) on the spherical harmonics basis. By construction, the $P_{N}$ approximation is a Friedrichs system. But simplifications for 2D flows leads to nonsymmetric systems. The $2 \mathrm{D}$ form of the $P_{N}$ equations (see [Bru02]-[BH05]-[Bru05]) is

$$
\left\{\begin{array}{l}
\frac{1}{c} \partial_{t} I_{l}^{m}+\frac{1}{2} \partial_{x}\left(-C_{l-1}^{m-1} I_{l-1}^{m-1}+D_{l+1}^{m-1} I_{l+1}^{m-1}+E_{l-1}^{m+1} I_{l-1}^{m+1}-F_{l+1}^{m+1} I_{l+1}^{m+1}\right) \\
+\partial_{z}\left(A_{l-1}^{m} I_{l-1}^{m}+B_{l+1}^{m} I_{l+1}^{m}\right)-\sigma I_{l}^{m}=0
\end{array}\right.
$$

for $m \neq 0$ and

$$
\left\{\begin{array}{l}
\frac{1}{c} \partial_{t} I_{l}^{0}+\frac{1}{2} \partial_{x}\left(E_{l-1}^{1} I_{l-1}^{1}+F_{l+1}^{1} I_{l+1}^{1}\right) \\
+\partial_{z}\left(A_{l-1}^{0} I_{l-1}^{0}+B_{l+1}^{0} I_{l+1}^{0}\right)+\sigma\left(I_{0}^{0} \delta_{l 0}-I_{l}^{0}\right)=0
\end{array}\right.
$$

for $m=0$. The coefficients are defined by

$$
\begin{gathered}
A_{l}^{m}=\sqrt{\frac{(l-m+1)(l+m+1)}{(2 l+3)(2 l+1)}} \quad B_{l}^{m}=\sqrt{\frac{(l-m)(l+m)}{(2 l+1)(2 l-1)},} \\
C_{l}^{m}=\sqrt{\frac{(l+m+1)(l+m+2)}{(2 l+3)(2 l+1)}} \quad D_{l}^{m}=\sqrt{\frac{(l-m)(l-m-1)}{(2 l+1)(2 l-1)}}, \\
E_{l}^{m}=\sqrt{\frac{(l-m+1)(l-m+2)}{(2 l+3)(2 l+1)}} \quad F_{l}^{m}=\sqrt{\frac{(l+m)(l+m-1)}{(2 l+1)(2 l-1)}},
\end{gathered}
$$

with $A_{l-1}^{m}=B_{l}^{m}, C_{l}^{m}=F_{l+1}^{m+1}$ and $D_{l}^{m}=E_{l-1}^{m+1}$. The system formed by the equations (44)-(45) is not symmetric. However, by an elementary change of unknowns we obtain a symmetric system. If we note $\tilde{I}_{l}^{m}$ the unknowns defined by:

- $\tilde{I}_{l}^{m}=I_{l}^{m}$,

- $\tilde{I}_{l}^{m}=-\sqrt{2} I_{l}^{m}$,

then the $P_{N}$ system associated to $\tilde{I}_{l}^{m}$ is symmetric.

The $P_{N}$ systems satisfy the following properties

- $R$ is a diagonal matrix. 0 is an eigenvalue with the multiplicity 1 and 1 is an eigenvalue with the multiplicity $n-1$ [Bru05]-[Bru02]. 
- The eigenvalues of the system are included in ] - 1,1[ [Bru05]-[Bru02].

- The hypothesis $\left(H_{2}\right)$ is verified (the spherical harmonics form a orthogonal basis for the $L^{2}$ scalar product).

In the numerical test, we use the $P_{3}$ model for which

$$
\begin{aligned}
& A_{1}=\left(\begin{array}{cccccccccc}
0 & \sqrt{\frac{1}{3}} & 0 & 0 & 0 & 0 & 0 & 0 & 0 & 0 \\
\sqrt{\frac{1}{3}} & 0 & \sqrt{\frac{4}{15}} & 0 & 0 & 0 & 0 & 0 & 0 & 0 \\
0 & \sqrt{\frac{4}{15}} & 0 & \sqrt{\frac{9}{35}} & 0 & 0 & 0 & 0 & 0 & 0 \\
0 & 0 & \sqrt{\frac{9}{35}} & 0 & 0 & 0 & 0 & 0 & 0 & 0 \\
0 & 0 & 0 & 0 & 0 & \sqrt{\frac{1}{5}} & 0 & 0 & 0 & 0 \\
0 & 0 & 0 & 0 & \sqrt{\frac{1}{5}} & 0 & \sqrt{\frac{8}{35}} & 0 & 0 & 0 \\
0 & 0 & 0 & 0 & 0 & \sqrt{\frac{8}{35}} & 0 & 0 & 0 & 0 \\
0 & 0 & 0 & 0 & 0 & 0 & 0 & 0 & \sqrt{\frac{1}{7}} & 0 \\
0 & 0 & 0 & 0 & 0 & 0 & 0 & \sqrt{\frac{1}{7}} & 0 & 0 \\
0 & 0 & 0 & 0 & 0 & 0 & 0 & 0 & 0 & 0
\end{array}\right), \\
& A_{2}=\left(\begin{array}{cccccccccc}
0 & 0 & 0 & 0 & \sqrt{\frac{1}{3}} & 0 & 0 & 0 & 0 & 0 \\
0 & 0 & 0 & 0 & 0 & \sqrt{\frac{1}{5}} & 0 & 0 & 0 & 0 \\
0 & 0 & 0 & 0 & -\sqrt{\frac{1}{15}} & 0 & \sqrt{\frac{6}{35}} & 0 & 0 & 0 \\
0 & 0 & 0 & 0 & 0 & -\sqrt{\frac{3}{35}} & 0 & 0 & 0 & 0 \\
\sqrt{\frac{1}{3}} & 0 & -\sqrt{\frac{1}{15}} & 0 & 0 & 0 & 0 & -\sqrt{\frac{1}{5}} & 0 & 0 \\
0 & \sqrt{\frac{1}{5}} & 0 & -\sqrt{\frac{3}{35}} & 0 & 0 & 0 & 0 & -\sqrt{\frac{1}{7}} & 0 \\
0 & 0 & \sqrt{\frac{6}{35}} & 0 & 0 & 0 & 0 & \sqrt{\frac{1}{70}} & 0 & 0 \\
0 & 0 & 0 & 0 & -\sqrt{\frac{1}{5}} & 0 & \sqrt{\frac{1}{70}} & 0 & 0 & -\sqrt{\frac{3}{14}} \\
0 & 0 & 0 & 0 & 0 & -\sqrt{\frac{1}{7}} & 0 & 0 & 0 & 0 \\
0 & 0 & 0 & 0 & 0 & 0 & 0 & -\sqrt{\frac{3}{14}} & 0 & 0
\end{array}\right) .
\end{aligned}
$$

Remark 5.1. Since the spherical harmonics are eigenvectors of scattering operators of the form

$$
Q(f)=\int_{S^{2}} p\left(\boldsymbol{\Omega}, \boldsymbol{\Omega}^{\prime}\right)\left(f\left(t, \mathbf{x}, \boldsymbol{\Omega}^{\prime}\right)-f(t, \mathbf{x}, \boldsymbol{\Omega})\right) d \boldsymbol{\Omega}^{\prime}
$$

or

$$
Q(f)=\triangle_{\boldsymbol{\Omega}} f(t, \mathbf{x}, \boldsymbol{\Omega}),
$$

where $\triangle_{\boldsymbol{\Omega}}$ is the Laplace-Beltrami operator defined on the sphere and $p\left(\boldsymbol{\Omega}, \boldsymbol{\Omega}^{\prime}\right)$ is an angular repartition function, therefore the "diffusive - non diffusive" decomposition for $P_{N}$ models associated to the transport equation with these operators is still valid since the assumption $\left(\mathrm{H}_{2}\right)$ is verified.

\section{Applications to the $S_{N}$ models}

The $S_{N}$ models for the transport equation (43) are defined by

$$
\partial_{t} f_{i}+\frac{1}{\varepsilon} \boldsymbol{\Omega}_{i} \cdot \nabla f_{i}=-\frac{\sigma}{\varepsilon^{2}}\left(f_{i}-\sum_{j} f_{j} w_{j}\right),
$$


with $f_{i}=f\left(\boldsymbol{\Omega}_{i}\right), \boldsymbol{\Omega}_{i}$ a discrete direction and $w_{i}$ and quadrature weight.

$$
\sum_{j} w_{j}=1, \sum_{j} w_{j} \boldsymbol{\Omega}_{j}=\mathbf{0}, \sum_{j} w_{j} \boldsymbol{\Omega}_{j} \otimes \boldsymbol{\Omega}_{j}=D_{c} \widehat{I}_{d}
$$

and $D_{c}=\frac{1}{3}$ if the velocities are defined in $\mathbb{S}^{2}$ and $D_{c}=\frac{1}{2}$ if the velocities are defined in $\mathbb{S}^{1}$. Usually the quadrature formula is symmetric with respect the rotation of the axis. These systems admit the following diffusion limit

$$
\partial_{t} E-\operatorname{div}(D \nabla E)=0
$$

with $E=\sum_{j} w_{j} f_{j}$ and $D=\frac{1}{\sigma} \sum_{j} w_{j} \boldsymbol{\Omega}_{j} \otimes \boldsymbol{\Omega}_{j}$.

Proposition 6.1. The $S_{N}$ models can be formulate to

$$
\partial_{t} \mathbf{U}+\frac{1}{\varepsilon} A_{1} \partial_{x} \mathbf{U}+\frac{1}{\varepsilon} A_{2} \partial_{y} \mathbf{U}=-\frac{\sigma}{\varepsilon^{2}} R \mathbf{U},
$$

with $U_{j}=\sqrt{w_{j}} f_{j}$ for each $j$ and $R=\widehat{I}_{d}-\sqrt{\mathbf{w}} \otimes \sqrt{\mathbf{w}}$. The vector $\sqrt{\mathbf{w}}$ is given by the the square root of $w_{j}$.

This system satisfies the following properties

- $\operatorname{dim} \operatorname{Ker} R=1$,

- $A_{1}$ and $A_{2}$ are diagonals,

- 0 is an eigenvalue of $R$ with the multiplicity 1 and the eigenvector $\mathbf{E}_{1}=\left(\sqrt{w_{1}}, \ldots ., \sqrt{w_{n}}\right)$,

- 1 is an eigenvalue of $R$ with the multiplicity $n-1$,

- The matrix $R$ is symmetric with real coefficients,

- $A_{1} \mathbf{E}_{1}=a \mathbf{E}_{2}, A_{2} \mathbf{E}_{1}=a \mathbf{E}_{3}$.

Proof. We first prove that 1 is an eigenvalue with the multiplicity $n-1$. We notice that $\widehat{I}_{d}-$ $\sqrt{\mathbf{w}} \otimes \sqrt{\mathbf{w}}$ corresponds to the orthogonal projection on the hyperplane orthogonal to the vector $\sqrt{\mathbf{w}}$. Therefore 1 is the eigenvalue of the matrix $R$ with the multiplicity $n-1$. The projection in the space generate by $\sqrt{\mathbf{w}}$ is equal to zero, thus 0 is an eigenvalue of $R$ associated to the eigenvector $\mathbf{E}_{1}=\sqrt{\mathbf{w}}$. In a second step, we show that the last property of the proposition 6.1 is verified. The condition under the quadrature point $\sum_{i} w_{i} \boldsymbol{\Omega}_{i}=\mathbf{0}$ imply that $\left(A_{1} \sqrt{\mathbf{w}}, \sqrt{\mathbf{w}}\right)=0$ and $\left(A_{2} \sqrt{\mathbf{w}}, \sqrt{\mathbf{w}}\right)=0$. Consequently $A \mathbf{E}_{1} \in \operatorname{Ker}(R)^{\perp}$ and $A \mathbf{E}_{2} \in \operatorname{Ker}(R)^{\perp}$. Using

$$
\mathbf{E}_{2}=\frac{1}{\sqrt{\sum_{i} w_{i} \Omega_{i}^{x, 2}}}\left(\begin{array}{c}
\Omega_{1}^{x} \\
\vdots \\
\Omega_{n}^{x}
\end{array}\right), \mathbf{E}_{3}=\frac{1}{\sqrt{\sum_{i} w_{i} \Omega_{i}^{y, 2}}}\left(\begin{array}{c}
\Omega_{1}^{y} \\
\vdots \\
\Omega_{n}^{y}
\end{array}\right) .
$$

we obtain $A_{1} \mathbf{E}_{1}=a \mathbf{E}_{2}, A_{2} \mathbf{E}_{1}=a \mathbf{E}_{3}$ with $a=\sqrt{\sum_{i} w_{i} \Omega_{i}^{x, 2}}=\sqrt{\sum_{i} w_{i} \Omega_{i}^{y, 2}}$. The equality $\sqrt{\sum_{i} w_{i} \Omega_{i}^{x, 2}}=\sqrt{\sum_{i} w_{i} \Omega_{i}^{y, 2}}$ comes from

$$
\sum_{i} w_{i} \boldsymbol{\Omega}_{i} \otimes \boldsymbol{\Omega}_{i}=D_{c} \widehat{I}_{d}
$$

with $D_{c}=\frac{1}{3}$ or $D_{c}=\frac{1}{2}$.

Remark 6.2. In dimension one, the $S_{N}$ models with Gauss-Legendre quadrature, are equivalent to the $P_{N}$ models.

Remark 6.3. Unlike the case of the $P_{N}$ model, the assumptions $\left(H_{2}\right)$ are not satisfied for the anisotropic scattering (49). 
The $S_{2}$ model used in the numerical examples writes

$$
\partial_{t} \mathbf{U}+\frac{1}{\varepsilon} A_{1} \partial_{x} \mathbf{U}+\frac{1}{\varepsilon} A_{2} \partial_{y} \mathbf{U}=-\frac{\sigma}{\varepsilon^{2}} R \mathbf{U}
$$

with

$$
A_{1}=\left(\begin{array}{cccc}
1 & 0 & 0 & 0 \\
0 & 0 & 0 & 0 \\
0 & 0 & -1 & 0 \\
0 & 0 & 0 & 0
\end{array}\right), \quad A_{2}=\left(\begin{array}{cccc}
0 & 0 & 0 & 0 \\
0 & 1 & 0 & 0 \\
0 & 0 & 0 & 0 \\
0 & 0 & 0 & -1
\end{array}\right), \quad R=\left(\begin{array}{cccc}
\frac{3}{4} & -\frac{1}{4} & -\frac{1}{4} & -\frac{1}{4} \\
-\frac{1}{4} & \frac{3}{4} & -\frac{1}{4} & -\frac{1}{4} \\
-\frac{1}{4} & -\frac{1}{4} & \frac{3}{4} & -\frac{1}{4} \\
-\frac{1}{4} & -\frac{1}{4} & -\frac{1}{4} & \frac{3}{4}
\end{array}\right)
$$

and the diffusion limit is

$$
\partial_{t} E-\operatorname{div}\left(\frac{1}{2 \sigma} \nabla E\right)=0
$$

with $E=\left(\frac{1}{4} \sum_{j} U_{j}\right)$. Defining the orthogonal matrix $Q$ and $D$ the diagonal matrix by

$$
Q=\left(\begin{array}{cccc}
\frac{1}{2} & \frac{1}{\sqrt{2}} & 0 & \frac{1}{2} \\
\frac{1}{2} & 0 & \frac{1}{\sqrt{2}} & -\frac{1}{2} \\
\frac{1}{2} & -\frac{1}{\sqrt{2}} & 0 & \frac{1}{2} \\
\frac{1}{2} & 0 & -\frac{1}{\sqrt{2}} & -\frac{1}{2}
\end{array}\right), \quad D=\left(\begin{array}{cccc}
0 & 0 & 0 & 0 \\
0 & 1 & 0 & 0 \\
0 & 0 & 1 & 0 \\
0 & 0 & 0 & 1
\end{array}\right)
$$

then in the unknowns $\mathbf{V}=Q^{t} \mathbf{U}$ the system writes

$$
\partial_{t} \mathbf{V}+\frac{1}{\varepsilon} A_{1}^{\prime} \partial_{x} \mathbf{V}+\frac{1}{\varepsilon} A_{2}^{\prime} \partial_{y} \mathbf{V}+=-\frac{\sigma}{\varepsilon^{2}} D \mathbf{V}
$$

with

$$
A_{1}^{\prime}=Q^{t} A_{1} Q=\left(\begin{array}{cccc}
0 & \frac{1}{\sqrt{2}} & 0 & 0 \\
\frac{1}{\sqrt{2}} & 0 & 0 & \frac{1}{\sqrt{2}} \\
0 & 0 & 0 & 0 \\
0 & \frac{1}{\sqrt{2}} & 0 & 0
\end{array}\right), \quad A_{2}^{\prime}=Q^{t} A_{2} Q=\left(\begin{array}{cccc}
0 & 0 & \frac{1}{\sqrt{2}} & 0 \\
0 & 0 & 0 & 0 \\
\frac{1}{\sqrt{2}} & 0 & 0 & -\frac{1}{\sqrt{2}} \\
0 & 0 & -\frac{1}{\sqrt{2}} & 0
\end{array}\right) .
$$

\section{$7 \quad$ Numerical results}

In this section we describe numerical results obtained for the three models, the hyperbolic heat equation (equivalent to $P_{1}$ ), $P_{3}$ and $S_{2}$ described previously. We give the results for both the diffusion and the transport regimes. For each model the results are obtained for 3 types of unstructured meshes as illustrated in figures 3 and 4. In this section, contour plots are for the first moment of the solution that is $\rho=\left(U, E_{1}\right)$ and we recall that $E_{1}$ is the basis of the kernel of $R$.
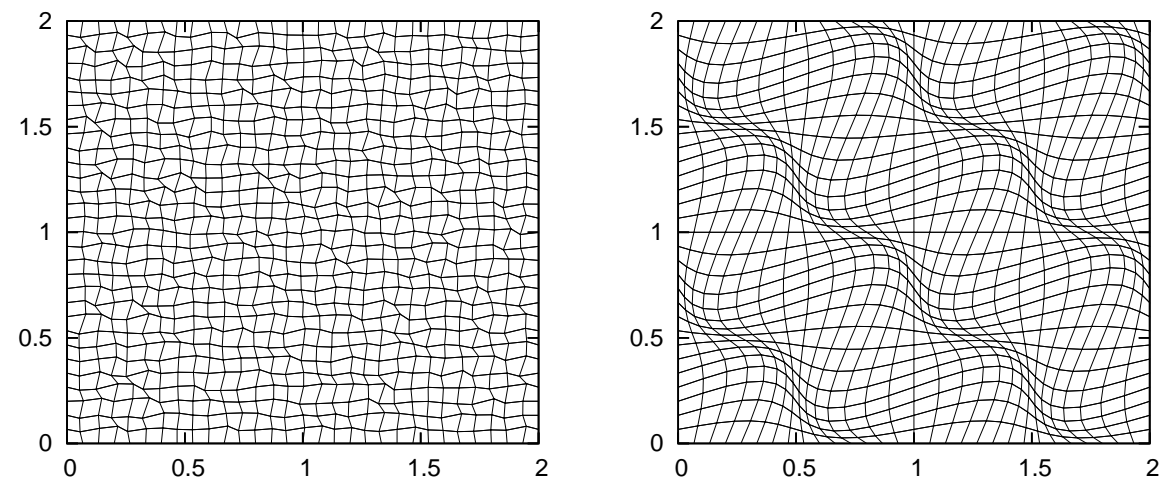

Figure 3: Unstructured quadrangular meshes 


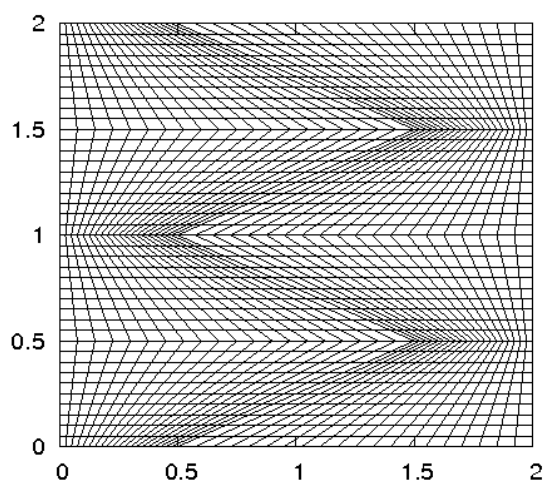

Figure 4: Kershaw mesh

\subsection{The $P_{1}$ model}

We illustrate the behavior of the asymptotic preserving discretization in $2 \mathrm{D}$. We use (24) to solve the $P_{1}$ model and we compare with an exact diffusion solution.

The test case is based on the fundamental solution of the heat equation with a diffusion coefficient equal to one, called $S F(t)$ [EGH00]. The initial datas are $U_{1}(t=0)=S F(0.01)$ and $U_{2}(t=0)=0$, $U_{3}(t=0)=0$. The diffusion solution is given by $U_{1}(t)=S(0.01+t), U_{2}(t)=0, U_{3}(t)=0$. We compare the exact diffusion solution, the solution obtained with the scheme without AP corrector, the solution obtained with the scheme with AP corrector which admits a non consistent TPFA diffusion scheme [BDF11] and the solution obtained with the consistent asymptotic preserving scheme (24). The exact diffusion solution is plotted on Cartesian mesh with 150 cells for each direction. The numerical solutions are computed on Kershaw mesh with 150 cells for each direction and $\varepsilon=0.0001$. When we solve this problem with the classic upwind scheme (fig. 5), we do
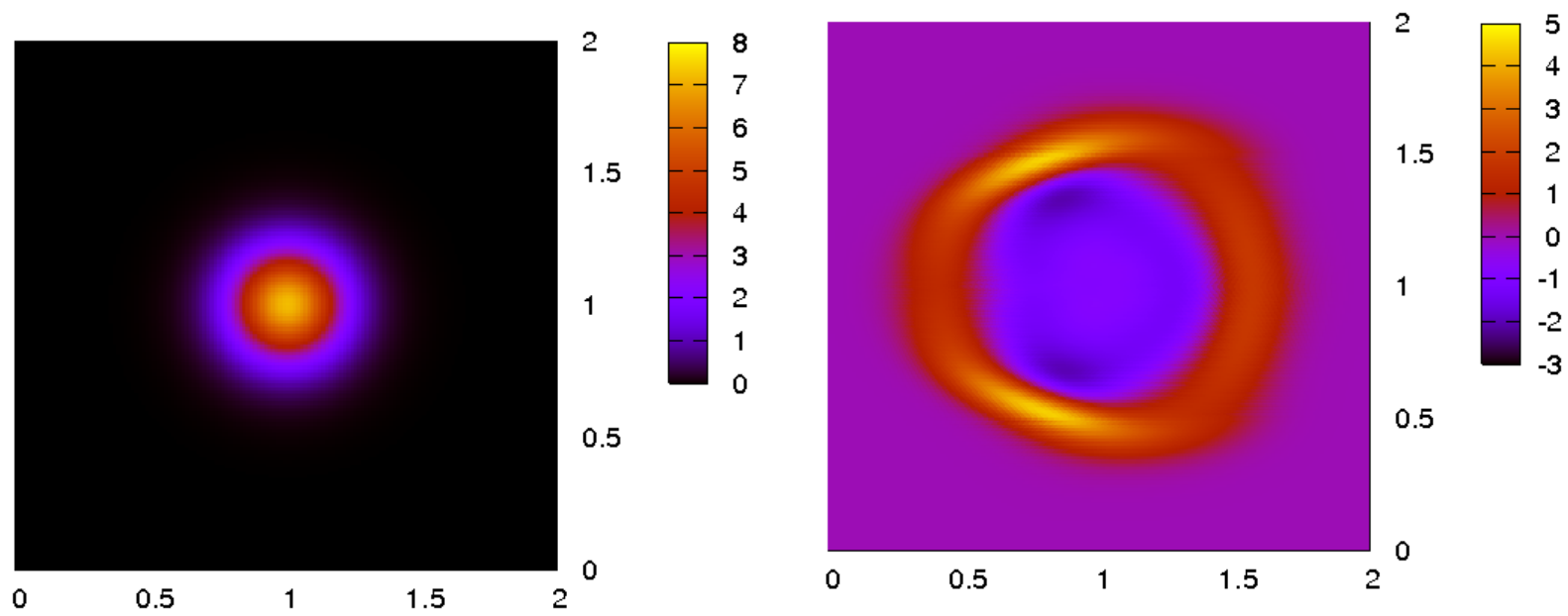

Figure 5: On the left, we plot the first moment $\rho$ of the exact solution of the test case at the time $t=0.01$. On the right, we plot $\rho$ obtained by the classical upwind scheme at the time $t=5 \times 10^{-5}$

not capture correctly the dynamic of the solution. For the TPFA-asymptotic preserving scheme (left on fig. 6), the quality of numerical solution is very dependent of the deformation of the mesh and the symmetry of the solution is not preserved. The numerical solution given by the nodal AP scheme is close to the exact solution. The quality of the numerical solution is not very sensitive to the mesh deformations. 

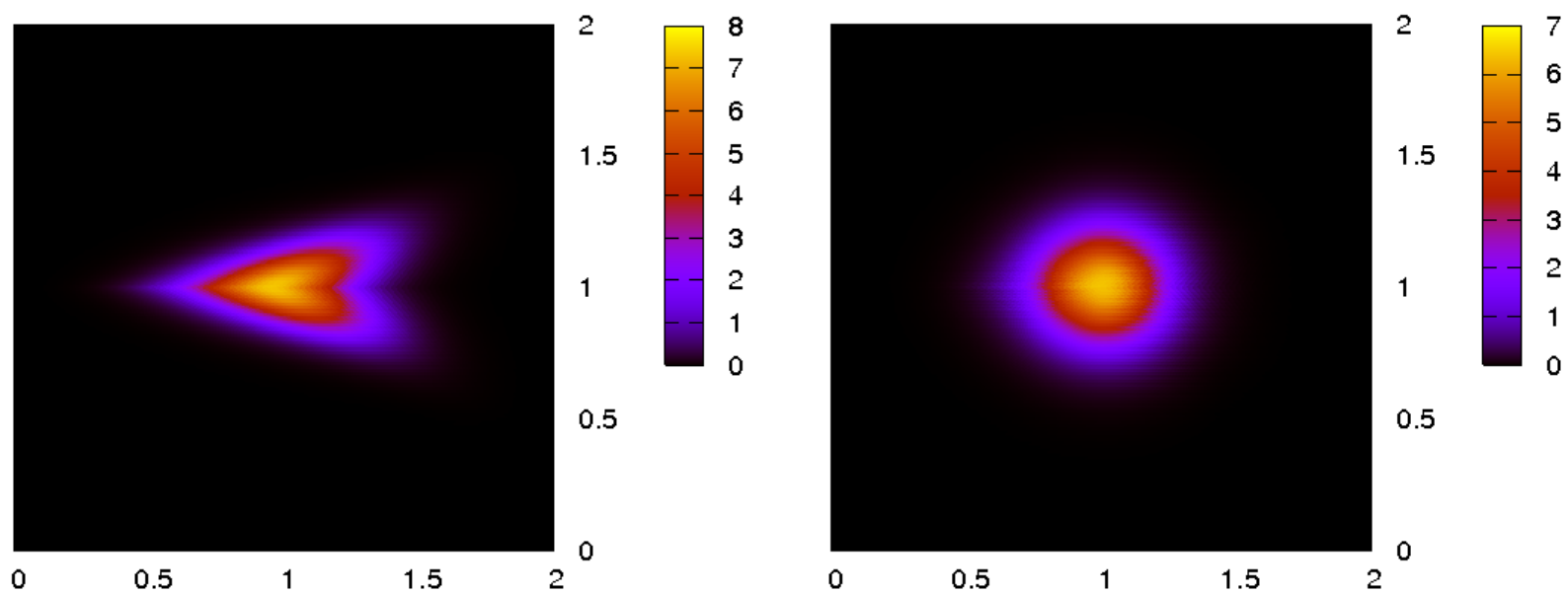

Figure 6: We plot the first moment $\rho$ of the solution at $t=0.01$ with two different schemes. In the left the scheme is a naive multidimensional extension of the usual 1-d AP scheme: this scheme is validated only on Delaunay meshes [BDF11]. In the right the scheme is the nodal JL-(b) (24-25) asymptotic preserving which is convergent on general meshes.

\subsection{The $S_{2}$ model}

We solve the Friedrichs $S_{2}$ system (2) with the matrices (52). The numerical scheme for the "diffusive" part is (24)-(25) and we use an implicit time discretization. We define the first moment $E=\sum_{i}^{4} w_{i} U_{i}$ with $\mathbf{U}=\left(U_{i=1}, \ldots, U_{i=4}\right)$.

\subsubsection{Numerical results in the diffusion regime}

We note $S F_{2}(t)$ the fundamental solution of the heat equation with a diffusion coefficient $\frac{1}{2}$. At the time zero, each unknown $U_{i}$ is equal at $S F_{2}(0.01)$. The solution at the time $T_{f}=0.01$ is $S F_{2}\left(0.01+T_{f}\right)$. The model is discretized with the JL-(b) nodal scheme for the "diffusive" part the upwind scheme for the other part and an implicit time discretization. We obtain the following results for the convergence.

\begin{tabular}{|c|c|c|c|c|}
\hline cells $/ \varepsilon$ & $\epsilon=10^{-3}$ & $\epsilon=10^{-4}$ & $\epsilon=10^{-6}$ & $\epsilon=10^{-7}$ \\
\hline \hline $40-80$ & 2.00 & 1.98 & 1.99 & 1.99 \\
\hline $80-160$ & 1.80 & 1.97 & 2. & 2 \\
\hline $160-320$ & 1.69 & 1.97 & 2.01 & 2.01 \\
\hline
\end{tabular}

Table 2: Order of convergence for the $S_{2}$ scheme on Cartesian mesh

\begin{tabular}{|c|c|c|c|c|}
\hline cells $/ \varepsilon$ & $\epsilon=10^{-3}$ & $\epsilon=10^{-4}$ & $\epsilon=10^{-6}$ & $\epsilon=10^{-7}$ \\
\hline \hline $40-80$ & 1.92 & 1.99 & 2.00 & 2.00 \\
\hline $80-160$ & 1.88 & 2.02 & 2.03 & 2.03 \\
\hline $160-320$ & 1.76 & 2.01 & 2.04 & 2.03 \\
\hline
\end{tabular}

Table 3: Order of convergence for the $S_{2}$ scheme on random quadrangular mesh

The tables (2)-(3)-(5)-(6)-(7) give the order of convergence for some meshes and values of $\varepsilon$. In the diffusion regime the numerical method converges with the second order.

These results deserve some remarks. The order of convergence for $\varepsilon=0.001$ and $\varepsilon=0.0001$ decreases a little when the number of cells increase. This phenomena comes from the fact that we 


\begin{tabular}{|c|c|c|c|c|}
\hline cells $/ \varepsilon$ & $\epsilon=10^{-3}$ & $\epsilon=10^{-4}$ & $\epsilon=10^{-6}$ & $\epsilon=10^{-7}$ \\
\hline \hline $40-80$ & 1.89 & 1.96 & 1.96 & 1.96 \\
\hline $80-160$ & 1.84 & 1.94 & 1.96 & 1.96 \\
\hline $160-320$ & 1.79 & 1.97 & 1.99 & 1.99 \\
\hline
\end{tabular}

Table 4: Order of convergence for the $S_{2}$ scheme on "smooth" mesh

\begin{tabular}{|c|c|c|c|c|}
\hline cells $/ \varepsilon$ & $\epsilon=10^{-3}$ & $\epsilon=10^{-4}$ & $\epsilon=10^{-6}$ & $\epsilon=10^{-7}$ \\
\hline \hline $40-80$ & 1.89 & 1.96 & 1.96 & 1.96 \\
\hline $80-160$ & 1.84 & 1.94 & 1.96 & 1.96 \\
\hline $160-320$ & 1.79 & 1.97 & 2.00 & 1.99 \\
\hline
\end{tabular}

Table 5: Order of convergence for the $S_{2}$ scheme on Kershaw mesh

\begin{tabular}{|c|c|c|c|c|}
\hline cells $/ \varepsilon$ & $\epsilon=10^{-3}$ & $\epsilon=10^{-4}$ & $\epsilon=10^{-6}$ & $\epsilon=10^{-7}$ \\
\hline \hline $40-80$ & 1.98 & 2.02 & 2.02 & 2.02 \\
\hline $80-160$ & 1.91 & 1.99 & 2.00 & 2.00 \\
\hline $160-320$ & 1.83 & 2.01 & 2.01 & 2.01 \\
\hline
\end{tabular}

Table 6: Order of convergence for the $S_{2}$ scheme on regular triangular mesh

\begin{tabular}{|c|c|c|c|c|}
\hline cells $/ \varepsilon$ & $\epsilon=10^{-3}$ & $\epsilon=10^{-4}$ & $\epsilon=10^{-6}$ & $\epsilon=10^{-7}$ \\
\hline \hline $40-80$ & 1.65 & 1.65 & 1.65 & 1.65 \\
\hline $80-160$ & 1.39 & 1.38 & 1.38 & 1.38 \\
\hline $160-320$ & 1.26 & 1.25 & 1.25 & 1.25 \\
\hline
\end{tabular}

Table 7: Order of convergence for the $S_{2}$ scheme on random triangular mesh

compare the numerical solution of the $S_{2}$ with the exact solution of the diffusion equation. But the exact diffusion is an approximation of the $S_{2}$ solution with an error homogeneous to $\varepsilon$. Therefore when the numerical error is close to $\varepsilon$, it is not justified to compare the error numerical with the diffusion solution.

\subsubsection{Transport test case}

We verify here that the "diffusive - non diffusive" decomposition and the AP corrector do not disturb the convergence in the transport regime.

\section{Test case 1}

It is a classical transport case. The quantities are initialized by $U_{1}=\chi_{[0.4,0.6]^{2}}$ and $U_{i}=0$ for $i>1$. We define $\sigma=0$ and $\varepsilon=1$. The solution for $U_{1}(t, \mathbf{x})$ is the initial solution advected with the velocity $(1,0)$, the other variables are equal to zero. The final time is $T_{f}=0.1$. Since the initial data is discontinuous the theoretical order is 0.5 for the norm $L^{1}$. We show the order for the variable $E=\sum_{i} w_{i} U_{i}$ in table 8 .

\section{Test case 2}

We note $G(\mathbf{x})$ a Gaussian function. The initial data are given by $U_{i}(\mathbf{x}, t=0)=G(\mathbf{x})$ and the parameters are defined by $\sigma=0$ and $\varepsilon=1$. The solution corresponds to the advection of four Gaussian functions with advection velocities $(0,1),(0,-1),(1,0)$ et $(-1,0)$. The final time is 0.2 . We compare the exact and numerical solutions for the quantity $E=\frac{1}{4} \sum_{i=1}^{4} U_{i}$. For this test case, the scheme converges with the first order as can seen on figure 7 . 


\begin{tabular}{|c|c|c|c|}
\hline Meshes order & $40-80$ & $80-160$ & $160-320$ \\
\hline Cartesian mesh & 0.45 & 0.48 & 0.51 \\
\hline Random quad. mesh & 0.47 & 0.48 & 0.50 \\
\hline Smooth mesh, & 0.47 & 0.46 & 0.47 \\
\hline Regular trig. mesh & 0.48 & 0.48 & 0.48 \\
\hline Random rig. mesh & 0.49 & 0.47 & 0.47 \\
\hline Kershaw mesh & 0.38 & 0.42 & 0.43 \\
\hline
\end{tabular}

Table 8: Order of convergence for the $S_{2}$ scheme for the test case 1
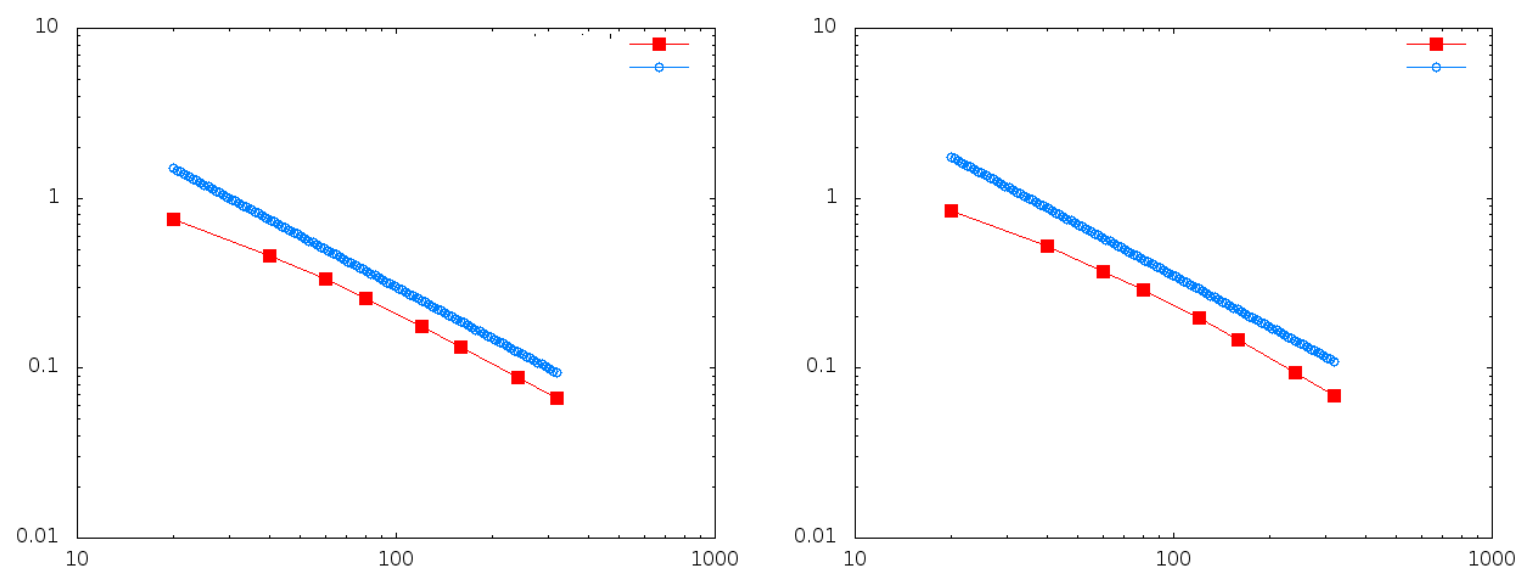

Figure 7: The red curve with square correspond to the numerical error on Cartesian mesh (left) and on random mesh (right). The blue curve with circle correspond to the function $\frac{1}{h}$.

\section{Test case 3:}

The initial data is $U_{i}=\delta_{1,1}$ with $\delta_{1,1}$ a Dirac function centered in $x=1$ and $y=1$. We take $\varepsilon=1$ and $\sigma=1$. The analytical solution is constructed with 4 Dirac functions advected in each direction. We use a random quadrangular mesh. The result is computed using the stabilized-nodal scheme (without spurious modes, see [BDF11]) for the "diffusive" part. The result is given by the figure (8).

Remark 7.1. The last test case allows to exhibit a default of the "diffusive-non diffusive" decomposition. Indeed the $S_{N}$ model preserves the positivity of the discrete distribution function associated to the linear kinetic equation, consequently all the unknowns are positive. This property is not preserve at the discrete level.

\subsection{The $P_{3}$ model}

In this subsection we validate our numerical method for the $P_{3}$ system. We verify the convergence in the diffusion limit. After we propose some test cases in the transport regime.

\subsubsection{Numerical results for $P_{3}$ in diffusion limit}

Let $S F_{3}(t)$ be the fundamental solution of the heat equation with a diffusion coefficient of $\frac{1}{3}$. The initial data is $U_{1}(t=0)=S F_{3}(0.01)$ and $U_{i}(t=0)=0$ for $i$ different of zero. The final time is $T_{f}=0.01$. The solution, at the final time, is the fundamental solution at $t=0.02$. We provide convergence order for implicit scheme and semi-implicit scheme obtained using the semi-implicit JL-(b) nodal scheme for the "diffusive" part and a modified Rusanov scheme for the other part (see subsection 4.2). The time step is given by $\Delta t=\frac{1}{2} h^{2}$ with $h$ the step mesh. 


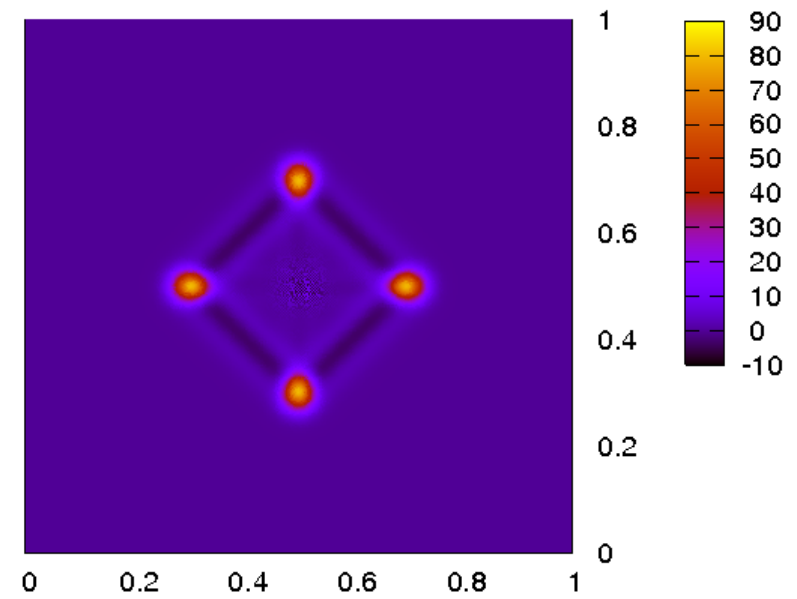

Figure 8: First moment $\rho$ of the fundamental solution of $S_{2}$ model

\begin{tabular}{|c||c|c|c|c|}
\hline Semi-implicit time discretization & \multicolumn{5}{|l|}{} \\
\hline \hline cells $/ \varepsilon$ & $\epsilon=10^{-3}$ & $\epsilon=10^{-4}$ & $\epsilon=10^{-6}$ & $\epsilon=10^{-7}$ \\
\hline $40-80$ & 1.91 & 2.01 & 2.02 & 2.02 \\
\hline $80-160$ & 1.81 & 1.98 & 2.00 & 2.00 \\
\hline $160-320$ & 1.66 & 1.96 & 2.00 & 2.00 \\
\hline \hline Implicit time discretization & \multicolumn{4}{|l}{} \\
\hline \hline cells $/ \varepsilon$ & $\epsilon=10^{-3}$ & $\epsilon=10^{-4}$ & $\epsilon=10^{-6}$ & $\epsilon=10^{-7}$ \\
\hline $40-80$ & 1.89 & 1.95 & 1.95 & 1.95 \\
\hline $80-160$ & 1.87 & 1.99 & 2.00 & 2.00 \\
\hline $160-320$ & 1.77 & 2.01 & 2.03 & 2.03 \\
\hline
\end{tabular}

Table 9: Order of convergence for the $P_{3}$ scheme on Cartesian mesh

\begin{tabular}{|c||c|c|c|c|}
\hline Semi-implicit time discretization & \multicolumn{4}{|l|}{} \\
\hline \hline cells $/ \varepsilon$ & $\epsilon=10^{-3}$ & $\epsilon=10^{-4}$ & $\epsilon=10^{-6}$ & $\epsilon=10^{-7}$ \\
\hline $40-80$ & 1.93 & 1.99 & 2.00 & 2.00 \\
\hline $80-160$ & 1.89 & 2.01 & 2.02 & 2.03 \\
\hline $160-320$ & 1.79 & 2.02 & 2.05 & 2.05 \\
\hline \hline Implicit time discretization & \multicolumn{4}{|l}{} \\
\hline \hline cells/ $\varepsilon$ & $\epsilon=10^{-3}$ & $\epsilon=10^{-4}$ & $\epsilon=10^{-6}$ & $\epsilon=10^{-7}$ \\
\hline $40-80$ & 1.89 & 1.95 & 1.95 & 1.95 \\
\hline $80-160$ & 1.86 & 1.99 & 2.00 & 2.00 \\
\hline $160-320$ & 1.77 & 2.01 & 2.03 & 2.03 \\
\hline
\end{tabular}

Table 10: Order of convergence for the $P_{3}$ scheme on random quadrangular mesh

The tables (9)-(10)-(12)-(13)-(14) give the order of convergence for some meshes and some values of $\varepsilon$. The remarks introduced on the convergence results of the asymptotic preserving scheme for the $S_{2}$ model are valid for this test case. 


\begin{tabular}{|c|c|c|c|c|}
\hline Semi-Implicit time discretization & \multicolumn{4}{|l|}{} \\
\hline \hline cells $/ \varepsilon$ & $\epsilon=10^{-3}$ & $\epsilon=10^{-4}$ & $\epsilon=10^{-6}$ & $\epsilon=10^{-7}$ \\
\hline $40-80$ & 2.03 & 2.1 & 2.11 & 2.11 \\
\hline $80-160$ & 1.89 & 2.02 & 2.03 & 2.03 \\
\hline $160-320$ & 1.76 & 1.98 & 2.01 & 2.01 \\
\hline \hline Implicit time discretization & \multicolumn{4}{|l}{} \\
\hline \hline cells $/ \varepsilon$ & $\epsilon=10^{-3}$ & $\epsilon=10^{-4}$ & $\epsilon=10^{-6}$ & $\epsilon=10^{-7}$ \\
\hline $40-80$ & 1.89 & 1.95 & 1.97 & 1.97 \\
\hline $80-160$ & 1.85 & 1.99 & 2.00 & 2.00 \\
\hline $160-320$ & 1.77 & 2.01 & 2.02 & 2.02 \\
\hline
\end{tabular}

Table 11: Order of convergence for the $P_{3}$ scheme on "smooth" mesh

\begin{tabular}{|c|c|c|c|c|}
\hline \multicolumn{1}{|c||}{ Semi-implicit time discretization } & \multicolumn{4}{|l|}{} \\
\hline \hline cells $/ \varepsilon$ & $\epsilon=10^{-3}$ & $\epsilon=10^{-4}$ & $\epsilon=10^{-6}$ & $\epsilon=10^{-7}$ \\
\hline $40-80$ & 1.89 & 1.93 & 1.93 & 1.93 \\
\hline $80-160$ & 1.87 & 1.96 & 1.95 & 1.95 \\
\hline $160-320$ & 1.83 & 1.97 & 1.99 & 1.99 \\
\hline \hline Implicit time discretization & \multicolumn{4}{|l}{} \\
\hline \hline cells $/ \varepsilon$ & $\epsilon=10^{-3}$ & $\epsilon=10^{-4}$ & $\epsilon=10^{-6}$ & $\epsilon=10^{-7}$ \\
\hline $40-80$ & 1.89 & 1.95 & 1.95 & 1.95 \\
\hline $80-160$ & 1.84 & 1.98 & 2.00 & 2.00 \\
\hline $160-320$ & 1.75 & 2.00 & 2.01 & 2.01 \\
\hline
\end{tabular}

Table 12: Order of convergence for the $P_{3}$ scheme on Kershaw mesh

\begin{tabular}{|c||c|c|c|c|}
\hline Semi-implicit time discretization & \multicolumn{4}{|l|}{} \\
\hline \hline cells $/ \varepsilon$ & $\epsilon=10^{-3}$ & $\epsilon=10^{-4}$ & $\epsilon=10^{-6}$ & $\epsilon=10^{-7}$ \\
\hline $40-80$ & 2.03 & 2.1 & 2.06 & 2.06 \\
\hline $80-160$ & 1.95 & 2.03 & 2.04 & 2.04 \\
\hline $160-320$ & 1.85 & 2.01 & 2.01 & 2.01 \\
\hline \hline Implicit time discretization & \multicolumn{4}{|l}{} \\
\hline \hline cells $/ \varepsilon$ & $\epsilon=10^{-3}$ & $\epsilon=10^{-4}$ & $\epsilon=10^{-6}$ & $\epsilon=10^{-7}$ \\
\hline $40-80$ & 1.93 & 1.96 & 1.95 & 1.95 \\
\hline $80-160$ & 1.87 & 1.99 & 2.00 & 2.00 \\
\hline $160-320$ & 1.80 & 2.01 & 2.03 & 2.03 \\
\hline
\end{tabular}

Table 13: Order of convergence for the $P_{3}$ scheme on regular triangular mesh

\subsubsection{Fundamental solution for $P_{3}$ and $P_{1}$ models}

Now we solve the $P_{3}$ and $P_{1}$ systems with $U_{1}(t=0)=\delta_{(1,1)}$ and $U_{i}(t=0)=0$ for $i \neq 1$ [FHSN11]. The "diffusive" part is approximated with the JL-(b) nodal scheme. The time discretization is implicit. This test case is described in [HMc11]. The final time is $T=1$. The exact solution is composed of Dirac functions with the velocities $\lambda_{i}\left(\lambda_{i}\right.$ are the eigenvalues of $\left.A_{1} n^{x}+A_{2} n^{y}\right)$ and smooth functions between the Dirac functions. At the beginning the smooth functions are non negatives and becomes negatives for large time. For the $P_{1}$ system, the speed wave is $\frac{1}{\sqrt{3}}$ and for the $P_{3}$ system the maximal velocity is approximately 0.86 . The numerical results reproduce this behavior, see figure 9 . 


\begin{tabular}{|c|c|c|c|c|}
\hline Semi-implicit time discretization & \multicolumn{4}{|l|}{} \\
\hline \hline cells / $\varepsilon$ & $\epsilon=10^{-3}$ & $\epsilon=10^{-4}$ & $\epsilon=10^{-6}$ & $\epsilon=10^{-7}$ \\
\hline $40-80$ & 1.95 & 1.97 & 1.98 & 1.98 \\
\hline $80-160$ & 1.89 & 1.99 & 2.01 & 2.01 \\
\hline $160-320$ & 1.81 & 2.00 & 2.02 & 2.02 \\
\hline \hline Implicit time discretization & \multicolumn{4}{|l}{} \\
\hline \hline cells/ $\varepsilon$ & $\epsilon=10^{-3}$ & $\epsilon=10^{-4}$ & $\epsilon=10^{-6}$ & $\epsilon=10^{-7}$ \\
\hline $40-80$ & 1.91 & 1.95 & 1.95 & 1.95 \\
\hline $80-160$ & 1.84 & 1.99 & 2.00 & 2.00 \\
\hline $160-320$ & 1.78 & 2.01 & 2.03 & 2.03 \\
\hline
\end{tabular}

Table 14: Order of convergence for the $P_{3}$ scheme on random triangular mesh
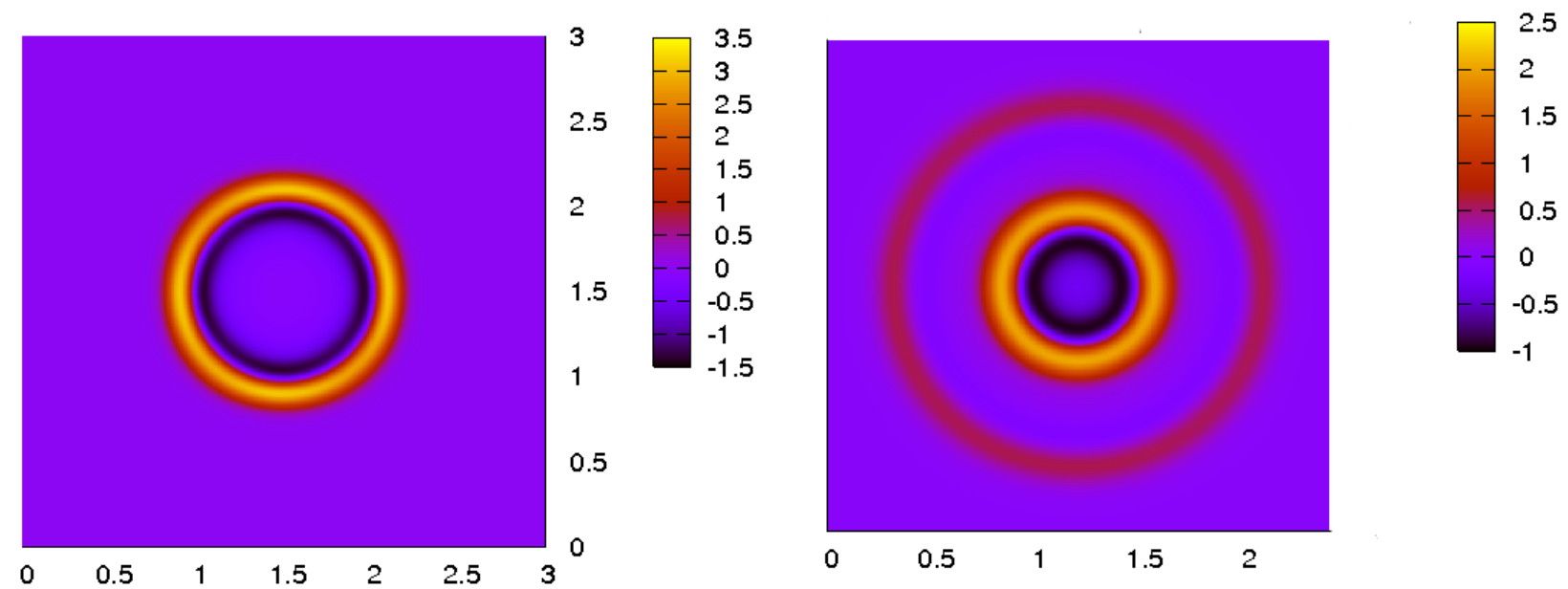

Figure 9: Left the first moment $\rho$ of the solution fundamental for the $P_{1}$ model at the time $T_{f}=1$, right $\rho$ for the fundamental solution for the $P_{3}$ model at the time $T_{f}=1$

\subsubsection{Lattice problem for $P_{3}$ and $P_{1}$ models}

This test case is an example of a complicated geometry. We consider a checker-board with different scattering and absorbing opacities on a lattice core (see [SFL11]). It is interesting for neutron transport simulations since is a simplification to a reactor core. The geometry is given in the figure 10. We define $\sigma$ the scattering opacity and $\sigma_{a}$ the absorption opacity. In the black square and the striped squares $\sigma_{a}=10$ and $\sigma=0$. In the white squares $\sigma=1$ and $\sigma_{a}=0$. The relaxation parameter is defined $\varepsilon=1$ in the whole domain. All the unknowns are equal to zero at the time 0 . We solve the $P_{1}$ and $P_{3}$ systems with the additional source term

$$
\partial_{t} \mathbf{U}+\frac{1}{\varepsilon} A \partial_{x} \mathbf{U}+\frac{1}{\varepsilon} B \partial_{x} \mathbf{U}=-\frac{\sigma}{\varepsilon^{2}} R \mathbf{U}+S
$$

where $A_{1}, A_{2}$ and $R$ are the matrices associated to the $P_{1}$ or $P_{3}$ system and $S_{i}=-\left(\sigma_{a} U_{i}+Q\right) \delta_{i 1}$ with $\delta_{i 1}$ a Kronecker product. The source $Q=1$ in the black square and $Q=0$ in the rest of the domain.

The $P_{3}$ systems is solved using the JL-(b) scheme for the "diffusive" part. We plot the first moment with a logarithmic scale $\log _{10}$ at the final time $T_{f}=3.2$. The results for $P_{1}$ and $P_{3}$ are given for the first moment in figures 11. They are the same as those in [SFL11]-[Bru02]. 


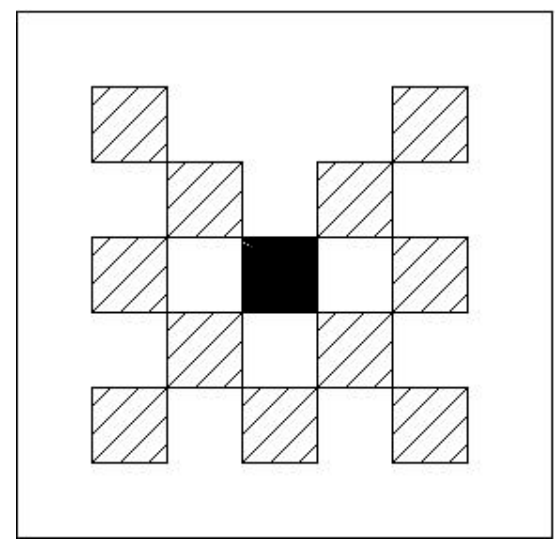

Figure 10: Geometry for the test case. the domain is $[0,7] \times[0,7]$
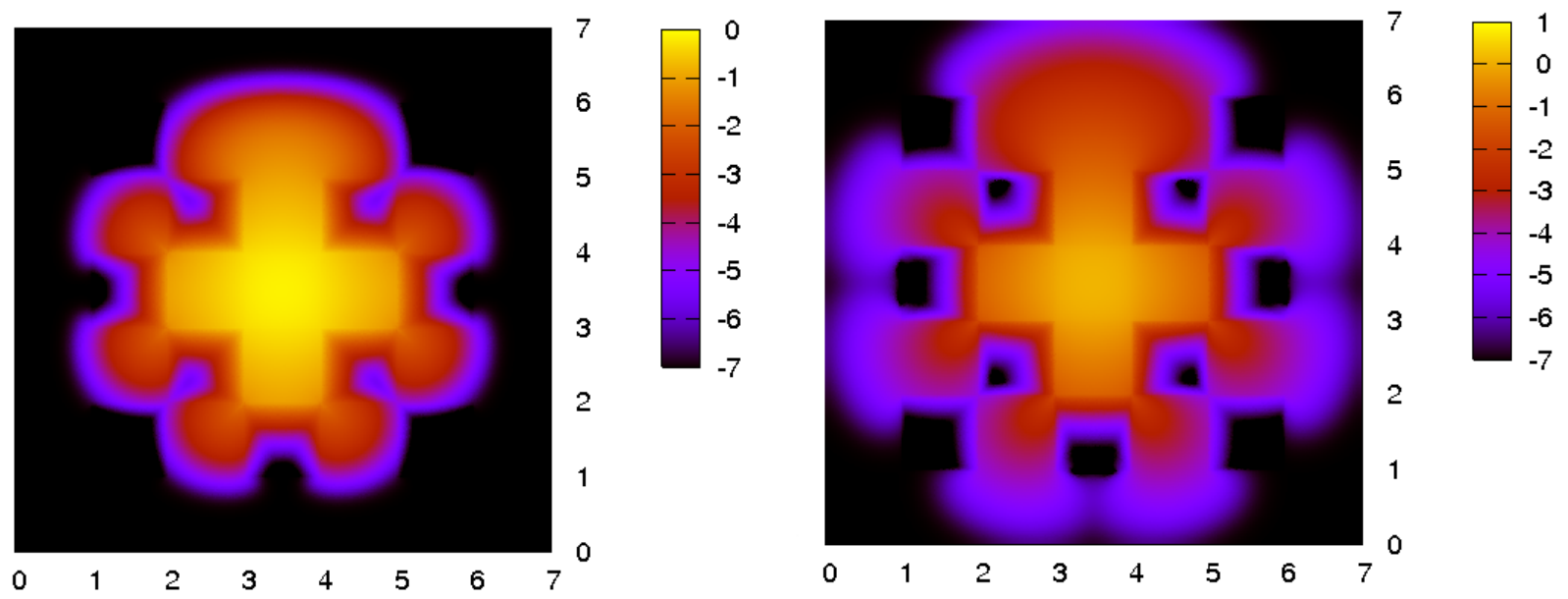

Figure 11: In the left, we solve the $P_{1}$ model and plot the $\log _{10}$ of the first moment $\rho$. In the right, we solve the $P_{3}$ model and plot the $\log _{10}$ of $\rho$.

\section{Conclusion}

We have studied the discretization on distorted meshes of linear hyperbolic systems with stiff source. We have proposed a method called "diffusive - non diffusive" decomposition which consists to split the hyperbolic system between the hyperbolic heat equation and a other system which is negligible in the diffusion regime. Using an asymptotic preserving scheme for the hyperbolic heat equation to discretize the "diffusive " part and a classical scheme to discretize the other part, we obtain an asymptotic preserving method for the complete system. For the approximation of transport equation, we use this decomposition for the simplified models as $P_{N}$ or $S_{N}$ approximations. For the $P_{N}$ systems the decomposition is natural. Since the first and second moments gives the limit regime. The others moments are close to $\varepsilon$. The high order moments are added only to obtain a better approximation in the pure transport regime $(\sigma=0)$. For the $S_{N}$ models, we remark that the diagonalized model admits a structure very close to the structure of the $P_{N}$ models. The "diffusive - non diffusive" decomposition gives consistent schemes for all the regimes. If the numerical methods used to discretize the different parts of the decomposition are stable in norm $L^{2}$, the method is stable in norm $L^{2}$. Modifying the schemes for the "non diffusive" part we can obtain a semi-implicit scheme with a CFL condition independent of $\varepsilon$. However this method is not optimal for the discretization of $S_{N}$ models, since our numerical method does not preserve 
the positivity. In the future, it would be interesting to design positive and asymptotic preserving method.

\section{References}

[AE06] I. Aavatsmark, G. Eigestad Numerical convergence of the MPFA O-method and U-method for general quadrilateral grids. Int J. Numer. Math. Fluids 2006; 51 939-961

[BCT08] C. Berthon, P. Charrier, R. Turpault An HLLC scheme to solve the $M^{1}$ model of radiative transfer in two dimensions J. Scie. Comput., J. Sci. Comput., 31, no. 3, pp. 347-389, 2007.

[B010] C. Berthon, J. Dubois, B. Dubroca, T.-H. Nguyen-Bui, R. Turpault, A free streaming contact preserving scheme for the $M_{1}$ model, Advances in Applied Mathematics and Mechanics Adv. Appl. Math. Mech., 3 (2010), pp. 259-285.

[BLeFT11] C. Berthon, P. LeFloch, R. Turpault Late-time relaxation limits of nonlinear hyperbolic systems. A general framework Math. of Comp.

[BT10] C. Berthon, R. Turpault Asymptotic preserving HLL schemes, Numerical Methods for Partial Differential Equations, 27 (6) (2011) pp 1396-1422.

[BCLM02] C. Buet, S. Cordier,B. Lucquin-Desreux, S. Mancini Diffusion limit of the lorentz model: asymptotic preserving schemes. ESAIM: M2AN vol. 36, 4, 2002, pp 631-655.

[BDF11] C. Buet, B. Desprès, E. Franck Design of asymptotic preserving schemes for the hyperbolic heat equation on unstructured meshes Numerish Mathematik, October 2012, Volume 122, Issue 2, pp 227-278.

[BDFCras] C. Buet, B. Després, E. Franck, An asymptotic preserving scheme with the maximum principle for the $M_{1}$ model on distorded meshes, C.R. Acad. Sci., Paris, Sér. I, Math., Vol 350, N 11-12 P. 633-638, juin 2012

[BDFproc] C. Buet, B. Després, E. Franck, Asymptotic preserving finite volumes discretization for non-linear moment model on unstructured meshes, Finite Volumes for Complex Applications VI Problems, Perspectives, Springer Proceedings in Mathematics Volume 4, 2011, pp 467-474

[BM06] J. Breil, P-H. Maire A cell-centered diffusion scheme on two-dimensional unstructured meshes. JCP 224 (2007) 785-823

[Bru05] T. Brunner Riemann solvers for time-dependant transport based on the maximum entropy and spherical harmonics closures. Ph.D thesis Los alamos.

[Bru02] T. Brunner Form of approximate radiation transport Sandia report, 2002.

[BH05] T. Brunner, J. P. Holloway Two-dimensional time dependent Riemann solvers for neutron transport JCP 210 386-399, 2005.

[CDDL09] G. Carré, S. Del Pino, B. Desprès, E. Labourasse A Cell-centered lagrangian hydrodynamics scheme on general unstructured meshes in arbitrary dimension. JCP vol. 228 (2009) no14, pp. 5160-518.

[CCGRS10] C. Chalons, F. Coquel, E. Godlewski, P. A. Raviart, N. Seguin Godunov-type schemes for hyperbolic systems with parameter-dependent source: the case of Euler system with friction, Math. Models Methods Appl. Sci. 20, 2109 (2010)

[Cou06] Y. Coudière, J. P. Vila, and P. Villedieu. Convergence d'un schéma volumes finis explicite en temps pour les systémes hyperboliques linéaires symétriques en domaines bornés. C.R. Acad. Sci., Paris, Sér. I, Math., 331:95-100, 2000. 
[CL11] N. Crouseilles, M. Lemou An asymptotic preserving scheme based on a micro-macro decomposition for collisional Vlasov equations: diffusion and high-field scaling limits, Kinetic and related models 4, 2 (2011) 441-477

[CL11] N. Crouseilles, M. Roger A dynamic multi-scale model for transient radiative transfer calculations Journal of Quantitative Spectroscopy and Radiative Transfer (2013)

[DW94] R. Dautray, J. P. Watteau, Fusion thermonucléaire inertielle par laser Eyrolles, 1994.

[DM04] D. Donatelli and P. Marcati Convergence of singular limits for multi-D semilinear hyperbolic systems to parabolic systems. Trans. Amer. Math. Soc. 356 (2004), no. 5, 2093-2121

[EGH00] L. C. Evans, Partial differential equations, second edition, Graduate Studies in Mathematics, American Mathematicals Society, 2010

[EGH00] R. Eymard, T. Gallouet, R. Herbin, The finite volume method, Handbook for Numerical Analysis, Ph. Ciarlet J.L. Lions eds, North Holland, 2000, 715-1022.

[FHSN11] E. Franck, P. Hoch, G. Samba, P. Navarro An asymptotic preserving scheme for $P_{1}$ model using classical diffusion schemes on unstructured polygonal meshes ESAIM: Proceedings, october 2011, Vol. 31, p. 56-75.

[GT01] L. Gosse, G. Toscani An asymptotic-preserving well-balanced scheme for the hyperbolic heat equations C. R. Acad. Sci Paris,Ser. I 334 (2002) 337-342.

[Goss11] L. Gosse Transient radiative transfer in the grey case: Well-balanced and asymptoticpreserving schemes built on Cases's elementary solutions Journal of Quantitative Spectroscopy and Radiative Transfer 112 (2011) 1995-2012.

[Goss12] L. Gosse Well-balanced schemes using elementary solutions for linear models of the Boltzmann equation in one space dimension Kinetic and related models 5, 2 (2012) 283 - 323.

[GL96] Greenberg J, Leroux A. Y. A well balanced scheme for the numerical processing of source terms in hyperbolic equations SIAM J. Numer. Anal - Vol 33 No 1, 1996.

[HMc11] C. D. Hauck, R. G McClarren Positive $P_{n}$ closures Siam, Journal on scientific computing, 2010

[HLMc10] C. D. Hauck, R. B. Lowrie, R. G McClarren Methods for diffusive relaxation in $P_{n}$ equations Numerical methods for relaxation systems and balance equations.

[Jin99] S. Jin, Efficient Asymptotic-Preserving (AP) Schemes for Some Multiscale Kinetic Equations, SIAM J. Sci. Comp. 21, 441-454, 1999

[Jin11] S. Jin, Asymptotic preserving (AP) schemes for multiscale kinetic and hyperbolic equations: a review, Lecture Notes for Summer School on "Methods and Models of Kinetic Theory" (M and MKT), Porto Ercole (Grosseto, Italy), Juin 2010.

[JL96] S. Jin, D. Levermore Numerical schemes for hyperbolic conservation laws with stiff relaxation terms. JCP 126,449-467, 1996.

[JL91] S. Jin, D. Levermore The discrete-ordinate method in diffusive regime. Transport Theory and Statistical Physics, vol. 20, issue 5, pp. 413-439, 1991

[KD10] G. Kluth, B. Després, Discretization of hyperelasticity on unstructured meshes with a cellcentered Lagrangian scheme J. Comp. Phys., 229, (2010), 9092-9118.

[LM07] M. Lemou, L.Mieussens A new asymptotic preserving scheme based on micro-macro formulation for linear kinetic equations inthe diffusion limit. SIAM J. Sci. COMPUT. Vol. 31,1, pp 334-368

[Mai11] P-H. Maire, Contribution to the numerical modeling of inertial confinement fusion, Hdr, CEA and university of Bordeaux. 
[SFL11] M. Schafer, M. Frank, C. D. Levermore, Diffusive corrections to $P_{n}$ approximations, Multiscale Model. Simul. 9 1-28, 2011. 